\title{
Intrinsic characterization of manifold-valued generalized functions
}

\author{
Michael Kunzinger * \\ Roland Steinbauer ${ }^{\dagger}$ \\ Department of Mathematics, University of Vienna \\ Strudlhofg. 4, A-1090 Wien, Austria \\ James A. Vickers $\ddagger$ \\ University of Southampton, Faculty of Mathematical Studies, \\ Highfield, Southampton SO17 1BJ, United Kingdom
}

April 2002

\begin{abstract}
The concept of generalized functions taking values in a differentiable manifold (15, 19) is extended to a functorial theory. We establish several characterization results which allow a global intrinsic formulation both of the theory of manifold-valued generalized functions and of generalized vector bundle homomorphisms. As a consequence, a characterization of equivalence that does not resort to derivatives (as provided in [11] for the scalar-valued cases of Colombeau's construction) is achieved. These results are employed to derive a point value description of all types of generalized functions valued in manifolds and to show that composition can be carried out unrestrictedly. Finally, a new concept of association adapted to the present setting is introduced.
\end{abstract}

Mathematics Subject Classification (2000): Primary: 46T30; secondary: 46F30, 53B20.

Keywords Algebras of generalized functions, Colombeau algebras, generalized functions on manifolds.

\section{Introduction}

While originating as a tool in the field of nonlinear PDEs a growing number of applications of nonlinear generalized functions , $4,5,21$ in a geometrical

\footnotetext{
*Electronic mail: michael.kunzinger@univie.ac.at

${ }^{\dagger}$ Electronic mail: roland.steinbauer@univie.ac.at

${ }_{\ddagger}^{\ddagger}$ Electronic mail: J.A.Vickers@maths.soton.ac.uk
} 
context - especially in the theory of general relativity (cf., e.g., 3, 2, 24, as well as 23] for a survey) and Lie group analysis of differential equations (cf. [16, 7, 12]) - has triggered the development of a geometric theory of algebras of generalized functions ([12]). Based on [1, 6, 14], a diffeomorphism invariant scalar theory in the so called "full" setting (which allows for a distinguished embedding of the space of Schwartz distributions) was developed in [11, 13. On the other hand the task of "geometrizing" the special setting of Colombeau's construction - which will also provide the framework for the present article - was started in [10, 8, 18, 19. In particular, in [18] a theory of generalized sections of vector bundles showing maximal compatibility with the distributional setting of [9. 20] was introduced. Also a point value description of generalized functions on differentiable manifolds was achieved. This setting in turn was used to set up a "generalized (pseudo-)Riemannian geometry" in [19]. Finally, in [15] the study of generalized functions taking values in a differentiable manifold was initiated. More precisely, the space $\mathcal{G}[X, Y]$ of generalized functions on the manifold $X$ and taking values in the manifold $Y$ was defined as was the space $\operatorname{Hom}_{\mathcal{G}}(E, F)$ of generalized vector bundle homomorphisms from $E$ to $F$. When dealing with generalized mappings on manifolds the need for such concepts arises quite naturally; for example when considering the flow of a generalized vector field as well as geodesics of a generalized metric.

The aim of the present paper is to complete this approach by extending it to a functorial theory. In particular, we provide several global characterizations of the notions of moderateness and equivalence (which replaces the notion of negligibility in the absence of a linear structure) for generalized functions from $X$ to $Y$ (Section 3) as well as for generalized vector bundle homomorphisms from $E$ to $F$ (Section (4). These characterizations in turn enable us to give a positive answer to the question raised in [15], Remark 2.11 whether equivalence is equivalent to equivalence of order zero. More precisely, we establish also in the case of both $\mathcal{G}[X, Y]$ and $\operatorname{Hom}_{\mathcal{G}}(E, F)$ a characterization of equivalence of nets without making any reference to derivatives provided the nets are assumed to be moderate (Theorems 3.3 and 4.2 ). Analogous results have been proven for virtually all variants of scalar- or vector valued Colombeau generalized functions (cf. [11], Th. 13.1, and [18], Sec. 4). In the context of $\mathcal{G}[X, Y]$ and $\operatorname{Hom}_{\mathcal{G}}(E, F)$ these characterizations provide the key to proving that (a) generalized functions taking values in a manifold as well as generalized vector bundle homomorphisms are characterized by their generalized point values (Theorem 3.5 respectively 4.5), and (b) composition of generalized functions between manifolds as well as of generalized vector bundle homomorphisms can be carried out unrestrictedly (Theorems 3.6 and 4.6). Analogous results are given in Section 5 for "hybrid" Colombeau spaces $\mathcal{G}^{h}[X, F]$ of generalized maps defined on a manifold $X$ and taking values in a vector bundle $F$ over $Y$. These spaces were introduced in [19], Sec. 4 to allow for the notion of generalized sections along generalized maps and, in particular, to define the notion of a geodesic of a generalized pseudoRiemannian metric. Moreover we prove that for any $u \in \mathcal{G}[X, Y]$ the space of generalized vector bundle homomorphisms on $u, \operatorname{Hom}_{u}(E, F)$ can be endowed with a natural vector space structure. While trivial in the smooth setting the 
corresponding result in the present framework relies on the fact that for any $v \in$ $\operatorname{Hom}_{u}(E, F)$ we may construct a representative whose induced map on the base coincides with a given representative of $u$ (Proposition 4.5). Finally in Section 6 we consider the concept of 'coupled calculus' and, in particular, the notion of zero-association which in the context of $\mathcal{G}[X, Y]$ allows us to make statements regarding compatibility of this approach with classical global analysis. We start this work by recalling the basic notions from the geometric theory of generalized functions (in the special setting) and, in particular, of generalized functions valued in a smooth manifold.

\section{Geometric theory of generalized functions}

We begin by fixing some notation. Throughout this paper $X$ and $Y$ denote smooth paracompact Hausdorff manifolds of dimension $n$ and $m$ respectively. Vector bundles with base space $X$ will be denoted by $(E, X, \pi)$ (or $\left(E, X, \pi_{X}\right)$ ) and for a chart $(V, \varphi)$ in $X$, a vector bundle chart $(V, \Phi)$ over $\varphi$ will be written in the form $(\mathbb{K}=\mathbb{R}$ respectively $\mathbb{C})$

$$
\begin{aligned}
\Phi: \pi^{-1}(V) & \rightarrow \varphi(V) \times \mathbb{K}^{n^{\prime}} \\
z & \rightarrow\left(\varphi(p), \varphi^{1}(z), \ldots, \varphi^{n^{\prime}}(z)\right) \equiv(\varphi(p), \varphi(z)),
\end{aligned}
$$

where $p=\pi(z)$ and the typical fiber is $\mathbb{K}^{n^{\prime}}$. Given a vector bundle atlas $\left(V_{\alpha}, \Phi_{\alpha}\right)_{\alpha}$ we write $\Phi_{\alpha} \circ \Phi_{\beta}^{-1}(y, w)=\left(\varphi_{\alpha \beta}(y), \varphi_{\boldsymbol{\alpha} \boldsymbol{\beta}}(y) w\right)$, where $\varphi_{\alpha \beta}:=\varphi_{\alpha} \circ \varphi_{\beta}^{-1}$ is the change of chart on the base and $\varphi_{\boldsymbol{\alpha} \beta}: \varphi_{\beta}\left(V_{\alpha} \cap V_{\beta}\right) \rightarrow \mathrm{GL}\left(n^{\prime}, \mathbb{K}\right)$ denotes the transition functions. The space of smooth sections of the vector bundle $(E, X, \pi)$ is denoted by $\Gamma(X, E)$, the space of smooth $(r, s)$-tensor fields (i.e., $\left.E=T_{s}^{r}\right)$ by $\mathcal{T}_{s}^{r}$ and the space of smooth vector bundle homomorphisms from $E$ to $F$ by $\operatorname{Hom}(E, F)$. If $f \in \operatorname{Hom}(E, F)$ we write $f: X \rightarrow Y$ for the smooth map induced on bases, i.e., $\pi_{Y} \circ f=f \circ \pi_{X}$. Local vector bundle homomorphisms with respect to vector bundle charts $(V, \Phi)$ of $E$ and $(W, \Psi)$ of $F$, i.e., $f_{\Psi \Phi}:=\Psi \circ f \circ \Phi^{-1}: \varphi\left(V \cap \underline{f}^{-1}(W)\right) \times \mathbb{K}^{n^{\prime}} \rightarrow \psi(W) \times \mathbb{K}^{m^{\prime}}$ will be written in the form

$$
f_{\Psi \Phi}(x, \xi)=\left(f_{\Psi \Phi}^{(1)}(x), f_{\Psi \Phi}^{(2)}(x) \cdot \xi\right) .
$$

Turning now to notions from Colombeau theory we set $I=(0,1]$ and $\mathcal{E}(X)=$ $\mathcal{C}^{\infty}(X)^{I}$ and let $\mathcal{P}(X)$ denote the space of linear differential operators on $X$. We define the spaces of moderate and negligible nets in $\mathcal{E}(X)$ by

$$
\begin{aligned}
\mathcal{E}_{M}(X):= & \left\{\left(u_{\varepsilon}\right)_{\varepsilon} \in \mathcal{E}(X): \forall K \subset \subset X, \forall P \in \mathcal{P}(X) \exists N \in \mathbb{N}:\right. \\
& \left.\sup _{p \in K}\left|P u_{\varepsilon}(p)\right|=O\left(\varepsilon^{-N}\right)\right\} \\
\mathcal{N}(X):= & \left.\left\{\left(u_{\varepsilon}\right)_{\varepsilon} \in \mathcal{E}_{M}(X): \forall K \subset \subset X, \forall q \in \mathbb{N}_{0}: \sup _{p \in K}\left|u_{\varepsilon}(p)\right|=O\left(\varepsilon^{q}\right)\right)\right\} .
\end{aligned}
$$

$\mathcal{G}(X):=\mathcal{E}_{M}(X) / \mathcal{N}(X)$ is called the special[ Colombeau algebra on $X$ and we

\footnotetext{
${ }^{1}$ Since we are going to work entirely in the "special" setting of Colombeau's construction we omit this term henceforth.
} 
denote its elements by $\left.u=\left[\left(u_{\varepsilon}\right)_{\varepsilon}\right)\right]$. $\mathcal{G}(-)$ is a fine sheaf of differential algebras with respect to the Lie derivative along smooth vector fields defined by $L_{\xi} u=$ $\left[\left(L_{\xi} u_{\varepsilon}\right)_{\varepsilon}\right] . u$ is in $\mathcal{G}(X)$ if and only if $u \circ \psi_{\alpha} \in \mathcal{G}\left(\psi_{\alpha}\left(V_{\alpha}\right)\right)$ (the local Colombeau algebra on $\left.\psi_{\alpha}\left(V_{\alpha}\right)\right)$ for all charts $\left(V_{\alpha}, \psi_{\alpha}\right)$. $\mathcal{C}^{\infty}(X)$ is a subalgebra of $\mathcal{G}(X)$ and there exist injective sheaf morphisms embedding $\mathcal{D}^{\prime}(X)$, the space of Schwartz distributions on $X$, into $\mathcal{G}(X)$.

A net $\left(p_{\varepsilon}\right)_{\varepsilon} \in X^{I}$ of points in $X$ is called compactly supported if $p_{\varepsilon}$ stays in a fixed compact set for small $\varepsilon$; the set of generalized points is denoted by $X_{c}$. Two nets $\left(p_{\varepsilon}\right),\left(q_{\varepsilon}\right)_{\varepsilon} \in X^{I}$ are called equivalent, $\left(p_{\varepsilon}\right)_{\varepsilon} \sim\left(q_{\varepsilon}\right)_{\varepsilon}$, if $d_{h}\left(p_{\varepsilon}, q_{\varepsilon}\right)=O\left(\varepsilon^{m}\right)$ for each $m>0$, where $d_{h}$ denotes the distance function induced on $X$ by any Riemannian metric $h$. The quotient space $\widetilde{X}_{c}$ of the set of compactly supported points modulo $\sim$ is called the space of compactly supported generalized points on $X$ and we write $\tilde{p}=\left[\left(p_{\varepsilon}\right)_{\varepsilon}\right]$. Inserting $\tilde{p}$ into $u \in \mathcal{G}(X)$ yields a well-defined element $\left[\left(u_{\varepsilon}\left(p_{\varepsilon}\right)\right)_{\varepsilon}\right]$ of $\mathcal{K}$, the space of generalized numbers (corresponding to $\mathbb{K}=\mathbb{R}$ respectively $\mathbb{C}$ and defined as the set of moderate nets of numbers $\left(r_{\varepsilon}\right)_{\varepsilon} \in \mathbb{K}^{I}$ with $\left|r_{\varepsilon}\right|=O\left(\varepsilon^{-N}\right)$ for some $N$ modulo negligible nets $\left|r_{\varepsilon}\right|=O\left(\varepsilon^{m}\right)$ for each $m$ ). Moreover, $u \in \mathcal{G}(X)$ is uniquely determined by its point values on $\widetilde{X}_{c}$, i.e., $u=v \Leftrightarrow u(\tilde{p})=v(\tilde{p}) \forall \tilde{p} \in \widetilde{X}_{c}([18]$, Th. 1).

The $\mathcal{G}(X)$-module $\Gamma_{\mathcal{G}}(X, E)$ of generalized sections in $E$ is defined using analogous asymptotic estimates with respect to the norm on the fibers induced by any Riemannian metric on $X$. Setting $\Gamma_{\mathcal{E}}(X, E)=\Gamma(X, E)^{I}$ and denoting by $\mathcal{P}(X, E)$ the space of linear differential operators on $\Gamma(X, E)$ we define

$$
\begin{aligned}
& \Gamma_{\mathcal{E}_{M}}(X, E):=\left\{\left(s_{\varepsilon}\right)_{\varepsilon} \in \Gamma_{\mathcal{E}}(X, E): \forall P \in \mathcal{P}(X, E) \forall K \subset \subset X \exists N \in \mathbb{N}:\right. \\
& \left.\sup _{p \in K}\left\|P u_{\varepsilon}(p)\right\|=O\left(\varepsilon^{-N}\right)\right\} \\
& \Gamma_{\mathcal{N}}(X, E):=\left\{\left(s_{\varepsilon}\right)_{\varepsilon} \in \Gamma_{\mathcal{E}_{M}}(X, E): \forall K \subset \subset X \forall m \in \mathbb{N}:\right. \\
& \left.\sup _{p \in K}\left\|u_{\varepsilon}(p)\right\|=O\left(\varepsilon^{m}\right)\right\},
\end{aligned}
$$

Finally $\Gamma_{\mathcal{G}}(X, E):=\Gamma_{\mathcal{E}_{M}}(X, E) / \Gamma_{\mathcal{N}}(X, E)$. Any $s=\left[\left(s_{\varepsilon}\right)_{\varepsilon}\right] \in \Gamma_{\mathcal{G}}(X, E)$ corresponds to a family $\left(s_{\alpha}\right)_{\alpha}=\left(\left(s_{\alpha}^{i}\right)_{\alpha}\right)_{i=1}^{n^{\prime}}$, where $s_{\alpha}$ is called the local expression of $s$ with its components $s_{\alpha}^{i}:=\Psi_{\alpha}^{i} \circ s \circ \psi_{\alpha}^{-1} \in \mathcal{G}\left(\psi_{\alpha}\left(V_{\alpha}\right)\right)\left(i=1, \ldots, n^{\prime}\right)$ satisfying $s_{\alpha}^{i}(x)=\left(\boldsymbol{\psi}_{\alpha \beta}\right)_{j}^{i}\left(\psi_{\beta} \circ \psi_{\alpha}^{-1}(x)\right) s_{\beta}^{j}\left(\psi_{\beta} \circ \psi_{\alpha}^{-1}(x)\right)$ for all $x \in \psi_{\alpha}\left(V_{\alpha} \cap V_{\beta}\right) . \Gamma_{\mathcal{G}}(-, E)$ is a fine sheaf of projective and finitely generated $\mathcal{G}(X)$-modules (18, Th. 5) and we have

$$
\Gamma_{\mathcal{G}}(X, E)=\mathcal{G}(X) \otimes \Gamma(X, E),
$$

where the tensor product is taken over $\mathcal{C}^{\infty}(X)([18]$, Th. 4).

For later reference we now explicitly recall the main definitions from 15. The space $\mathcal{G}[X, Y]$ of compactly bounded (c-bounded) generalized Colombeau functions from $X$ to $Y$ is defined as the quotient of the set of $\mathcal{E}_{M}[X, Y]$ of moderate, c-bounded maps from $X$ to $Y$ by a certain equivalence relation defined below.

2.1 Definition. $\mathcal{E}_{M}[X, Y]$ is defined as the set of all $\left(u_{\varepsilon}\right)_{\varepsilon} \in \mathcal{C}^{\infty}(X, Y)^{I}$ satisfying 
(i) $\forall K \subset \subset \Omega \exists \varepsilon_{0}>0 \exists K^{\prime} \subset \subset Y \forall \varepsilon<\varepsilon_{0}: u_{\varepsilon}(K) \subseteq K^{\prime}$.

(ii) $\forall k \in \mathbb{N}$, for each chart $(V, \varphi)$ in $X$, each chart $(W, \psi)$ in $Y$, each $L \subset \subset V$ and each $L^{\prime} \subset \subset W$ there exists $N \in \mathbb{N}$ with

$$
\sup _{x \in L \cap u_{\varepsilon}^{-1}\left(L^{\prime}\right)}\left\|D^{(k)}\left(\psi \circ u_{\varepsilon} \circ \varphi^{-1}\right)(\varphi(p))\right\|=O\left(\varepsilon^{-N}\right) .
$$

2.2 Definition. $\left(u_{\varepsilon}\right)_{\varepsilon}$ and $\left(v_{\varepsilon}\right)_{\varepsilon} \in \mathcal{E}_{M}[X, Y]$ are called equivalent, $\left(u_{\varepsilon}\right)_{\varepsilon} \sim$ $\left(v_{\varepsilon}\right)_{\varepsilon}$, if the following conditions are satisfied:

(i) For all $K \subset \subset X, \sup _{p \in K} d_{h}\left(u_{\varepsilon}(p), v_{\varepsilon}(p)\right) \rightarrow 0(\varepsilon \rightarrow 0)$ for some (hence every) Riemannian metric $h$ on $Y$.

(ii) $\forall k \in \mathbb{N}_{0} \forall m \in \mathbb{N}$, for each chart $(V, \varphi)$ in $X$, each chart $(W, \psi)$ in $Y$, each $L \subset \subset V$ and each $L^{\prime} \subset \subset W$ :

$$
\sup _{x \in L \cap u_{\varepsilon}^{-1}\left(L^{\prime}\right) \cap v_{\varepsilon}^{-1}\left(L^{\prime}\right)}\left\|D^{(k)}\left(\psi \circ u_{\varepsilon} \circ \varphi^{-1}-\psi \circ v_{\varepsilon} \circ \varphi^{-1}\right)(\varphi(p))\right\|=O\left(\varepsilon^{m}\right) .
$$

Due to the c-boundedness condition (i.e., (i) in Definition 2.1) elements $u$ of $\mathcal{G}[X, Y]$ can model jump discontinuities but not $\delta$-like singularities; the latter, however, will arise as tangent maps of such $u$ (see below, a list of examples is provided by [15], 2.8). Inserting a compactly supported point $\tilde{p} \in \widetilde{X}_{c}$ into $u \in \mathcal{G}[X, Y]$ yields a well-defined element $\left[u_{\varepsilon}\left(p_{\varepsilon}\right)_{\varepsilon}\right] \in \widetilde{Y}_{c}$. However, $p \in \widetilde{X}_{c}$ characterize $u \in \mathcal{G}[X, Y]$ only up to equivalence of order zero $\left(\sim_{0}\right.$, to be defined below), i.e., $u(\tilde{p})=v(\tilde{p}) \forall \tilde{p} \Leftrightarrow\left(u_{\varepsilon}\right)_{\varepsilon} \sim_{0}\left(v_{\varepsilon}\right)_{\varepsilon}$ (Prop. 2.14 in 15]).

2.3 Definition. We call two elements $\left(u_{\varepsilon}\right)_{\varepsilon},\left(v_{\varepsilon}\right)_{\varepsilon}$ of $\mathcal{E}_{M}[X, Y]$ equivalent of order $0,\left(u_{\varepsilon}\right)_{\varepsilon} \sim_{0}\left(v_{\varepsilon}\right)_{\varepsilon}$ if they satisfy Definition 2.8 (i) and (ii) for $k=0$.

We now turn to the definition of generalized vector bundle homomorphisms by first introducing the respective notions of moderateness and equivalence.

2.4 Definition. $\mathcal{E}_{M}{ }^{\mathrm{VB}}[E, F]$ is the set of all $\left(u_{\varepsilon}\right)_{\varepsilon} \in \operatorname{Hom}(E, F)^{I}$ satisfying

(i) $\left(\underline{u_{\varepsilon}}\right)_{\varepsilon} \in \mathcal{E}_{M}[X, Y]$.

(ii) $\forall k \in \mathbb{N}_{0} \forall(V, \Phi)$ vector bundle chart in $E, \forall(W, \Psi)$ vector bundle chart in $F, \forall L \subset \subset V \forall L^{\prime} \subset \subset W \exists N \in \mathbb{N} \exists \varepsilon_{1}>0 \exists C>0$ with

$$
\left\|D^{(k)}\left(u_{\varepsilon \Psi \Phi}^{(2)}(\varphi(p))\right)\right\| \leq C \varepsilon^{-N}
$$

for all $\varepsilon<\varepsilon_{1}$ and all $p \in L \cap{\underline{u_{\varepsilon}}}^{-1}\left(L^{\prime}\right)$, where $\|\cdot\|$ denotes any matrix norm.

2.5 Definition. $\left(u_{\varepsilon}\right)_{\varepsilon},\left(v_{\varepsilon}\right)_{\varepsilon} \in \mathcal{E}_{M}{ }^{\mathrm{VB}}[E, F]$ are called vb-equivalent, $\left(\left(u_{\varepsilon}\right)_{\varepsilon} \sim_{v b}\right.$ $\left.\left(v_{\varepsilon}\right)_{\varepsilon}\right)$ if 
(i) $\left(\underline{u_{\varepsilon}}\right)_{\varepsilon} \sim\left(\underline{v_{\varepsilon}}\right)_{\varepsilon}$ in $\mathcal{E}_{M}[X, Y]$.

(ii) $\forall k \in \mathbb{N}_{0} \forall m \in \mathbb{N} \forall(V, \Phi)$ vector bundle chart in $E, \forall(W, \Psi)$ vector bundle chart in $F, \forall L \subset \subset V \forall L^{\prime} \subset \subset W \exists \varepsilon_{1}>0 \exists C>0$ such that:

$$
\left\|D^{(k)}\left(u_{\varepsilon \Psi \Phi}^{(2)}-v_{\varepsilon \Psi \Phi}^{(2)}\right)(\varphi(p))\right\| \leq C \varepsilon^{m}
$$

for all $\varepsilon<\varepsilon_{1}$ and all $p \in L \cap \underline{u}_{\varepsilon}^{-1}\left(L^{\prime}\right) \cap \underline{v}_{\varepsilon}^{-1}\left(L^{\prime}\right)$.

We now set $\operatorname{Hom}_{\mathcal{G}}[E, F]:=\mathcal{E}_{M}{ }^{\mathrm{VB}}[E, F] / \sim_{v b}$. For $u \in \operatorname{Hom}_{\mathcal{G}}[E, F], \underline{u}:=\left[\left(\underline{u}_{\varepsilon}\right)_{\varepsilon}\right]$ is a well-defined element of $\mathcal{G}[X, Y]$ uniquely characterized by $\underline{u} \circ \pi_{X}=\pi_{Y} \circ u$. The tangent map $T u:=\left[\left(T u_{\varepsilon}\right)_{\varepsilon}\right]$ of any $u \in \mathcal{G}[X, Y]$ is a well-defined element of $\mathrm{Hom}_{\mathcal{G}}[T X, T Y]$.

Analogously to the case of manifold valued nets of functions we will need the weaker notion of $v b$-0-equivalence, denoted $\sim_{v b 0}$, and defined by $\left(u_{\varepsilon}\right)_{\varepsilon} \sim_{v b 0}\left(v_{\varepsilon}\right)_{\varepsilon}$, if $\left(\underline{u_{\varepsilon}}\right)_{\varepsilon} \sim_{0}\left(\underline{v_{\varepsilon}}\right)_{\varepsilon}$ and (ii) above holds for $k=0$.

Finally we define the space $E_{c}^{\sim_{v b}}$ of compactly bounded generalized vector bundle points.

2.6 Definition. On $(E, X, \pi)$ we define the set of vb-moderate generalized points as consisting of $\left(e_{\varepsilon}\right)_{\varepsilon} \in E^{I}$ satisfying

(i) $\exists K \subset \subset X \exists \varepsilon_{0}>0$ such that $\left(\pi\left(e_{\varepsilon}\right)\right)_{\varepsilon} \in K$ for all $\varepsilon<\varepsilon_{0}$.

(ii) $\exists \varepsilon_{1}>0 \exists N \in \mathbb{N} \exists C>0$ such that $\left\|e_{\varepsilon}\right\| \leq C \varepsilon^{-N}$ for all $\varepsilon<\varepsilon_{1}$ (with the norm again induced by any Riemannian metric on the base).

On this set we introduce the equivalence relation $\left(e_{\varepsilon}\right)_{\varepsilon} \sim_{v b}\left(e_{\varepsilon}^{\prime}\right)_{\varepsilon}$ by

(iii) $\left(\pi\left(e_{\varepsilon}\right)\right)_{\varepsilon} \sim\left(\pi\left(e_{\varepsilon}^{\prime}\right)\right)_{\varepsilon}$ in $X^{I}$

(iv) $\forall m \in \mathbb{N} \forall(W, \Psi)$ vector bundle chart in $E \forall L^{\prime} \subset \subset W \exists \varepsilon_{1}>0 \exists C>0$ such that $\left|\boldsymbol{\psi} e_{\varepsilon}-\boldsymbol{\psi} e_{\varepsilon}^{\prime}\right| \leq C \varepsilon^{m}$ for all $\varepsilon<\varepsilon_{1}$ whenever both $\pi_{X}\left(e_{\varepsilon}\right)$ and $\pi_{X}\left(e_{\varepsilon}^{\prime}\right)$ lie in $L^{\prime}$. (Here $\Psi=\left(z_{p} \mapsto(\psi(p), \psi(z))\right)$, cf. (因).)

The set of equivalence classes is denoted by $E_{c}^{\sim v b}$.

Inserting $\tilde{p} \in \widetilde{X}_{c}$ into $u \in \Gamma_{\mathcal{G}}(X, E)$ yields a well-defined element of $E_{c}^{\sim v b}$ and generalized sections are characterized by these point values (15], Th. 3.7 (i)). On the other hand $v \in \operatorname{Hom}_{\mathcal{G}}[E, F]$ has to satisfy an additional condition (equation (6) in 15]) to allow for a well defined element $\left[\left(v_{\varepsilon}\left(e_{\varepsilon}\right)\right)_{\varepsilon}\right] \in F_{c}^{\sim_{v b}}$ for $\tilde{e} \in E_{c}^{\sim{ }^{v b}}$ (【15], Prop. 3.6 (ii)) and even under this restriction generalized vector

bundle homomorphisms are characterized by their point values on $E_{c}^{\sim v b}$ only up to $v b$-0-equivalence (15], Th. 3.7 (ii)).

\section{Characterization of generalized functions valued in a manifold}

We start this section by establishing simple and global criteria for nets $\left(u_{\varepsilon}\right)_{\varepsilon} \in$ $\mathcal{C}^{\infty}(X, Y)^{I}$ to be moderate. The basic idea is to replace the chartwise (in $Y$ ) 
description of Definition 2.1 (ii) by composition with smooth (compactly supported) functions $f: Y \rightarrow \mathbb{C}$. To begin with, we note the following characterization of the notion of c-boundedness.

3.1 Proposition. Let $\left(u_{\varepsilon}\right)_{\varepsilon} \in \mathcal{C}^{\infty}(X, Y)$. The following conditions are equivalent

(i) $\left(u_{\varepsilon}\right)_{\varepsilon}$ is c-bounded.

(ii) $\left(f \circ u_{\varepsilon}\right)_{\varepsilon}$ is c-bounded for all $f \in \mathcal{C}^{\infty}(Y)$.

(iii) $\left(f \circ u_{\varepsilon}\right)_{\varepsilon}$ is moderate of order zero for all $f \in \mathcal{C}^{\infty}(Y)$, i.e.,

$$
\forall K \subset \subset X \exists N \in \mathbb{N}: \sup _{p \in K}\left|f \circ u_{\varepsilon}(p)\right|=O\left(\varepsilon^{-N}\right)
$$

for all $f \in \mathcal{C}^{\infty}(Y)$.

(iv) $\left(u_{\varepsilon}\left(x_{\varepsilon}\right)\right)_{\varepsilon} \in Y_{c}$ for all $\left(x_{\varepsilon}\right)_{\varepsilon} \in X_{c}$.

Proof. (i) $\Rightarrow($ ii $) \Rightarrow($ iii) is clear and so is (i) $\Leftrightarrow($ iv). We establish (iii) $\Rightarrow$ (i). To begin with suppose $Y$ is non compact and connected. We then may write $Y=\bigcup_{n \in \mathbb{N}} L_{n}$ with $L_{n}$ compact and $L_{n}$ contained in the interior $L_{n+1}^{\circ}$ of $L_{n+1}$ for all $n$. Suppose (i) is false; then $\exists K \subset \subset X \forall n \in \mathbb{N} \exists \varepsilon_{n} \leq 1 / n \exists p_{n} \in K$ with $u_{\varepsilon_{n}}\left(p_{n}\right) \notin L_{n}$. Without loss of generality we may even suppose that $u_{\varepsilon_{n}}\left(p_{n}\right) \in L_{n+1}^{\circ} \backslash L_{n}$. Now choose $f_{n}$ in $\mathcal{D}\left(L_{n+1}^{\circ} \backslash L_{n}\right)$ with $f_{n}\left(u_{\varepsilon_{n}}\left(p_{n}\right)\right)=e^{1 / \varepsilon_{n}}$ and let $f:=\sum_{n=1}^{\infty} f_{n}$. Then $f \in \mathcal{C}^{\infty}(Y)$ and by (iii) we are guaranteed the existence of $N \in \mathbb{N}$ such that $\left|\sup _{p \in K} f\left(u_{\varepsilon}(p)\right)\right| \leq \varepsilon^{-N}$. Hence $\left|f\left(u_{\varepsilon_{n}}\left(p_{n}\right)\right)\right| \leq \varepsilon_{n}^{-N}$ but by the above $e^{1 / \varepsilon_{n}} \leq \varepsilon_{n}^{-N}$ for large $n$, a contradiction. If $Y$ is not connected we employ a similar construction taking into account that each connected component of $Y$ is $\sigma$-compact.

We now have the following characterization of moderateness in $\mathcal{C}^{\infty}(X, Y)^{I}$.

3.2 Proposition. Let $\left(u_{\varepsilon}\right)_{\varepsilon} \in \mathcal{C}^{\infty}(X, Y)^{I}$. The following statements are equivalent.

(a) $\left(u_{\varepsilon}\right)_{\varepsilon} \in \mathcal{E}_{M}[X, Y]$.

(b) (i) $\left(u_{\varepsilon}\right)_{\varepsilon}$ is c-bounded.

(ii) $\left(f \circ u_{\varepsilon}\right)_{\varepsilon} \in \mathcal{E}_{M}(X)$ for all $f \in \mathcal{D}(Y)$.

(c) $\left(f \circ u_{\varepsilon}\right)_{\varepsilon} \in \mathcal{E}_{M}(X)$ for all $f \in \mathcal{C}^{\infty}(Y)$.

Note that (i) in (b) is necessary. Indeed, let $X=\mathbb{R}=Y$; then $u_{\varepsilon}(x)=1 / \varepsilon$ is not c-bounded but $\left|f \circ u_{\varepsilon}(x)\right| \leq\|f\|_{\infty}$ for all compactly supported smooth $f$.

Proof. (b) $\Rightarrow($ a): We have to verify (i) and (ii) of Definition 2.1. (i) is identical to (b) (i). Concerning (ii), let $k \in \mathbb{N},(V, \varphi),(W, \psi)$ charts in $X$ respectively $Y$ 
and $L \subset \subset V, L^{\prime} \subset \subset W$. Choose $f \in \mathcal{D}(W)^{m}, f \equiv \psi$ in a neighborhood of $L^{\prime}$ and set $f_{j}:=\operatorname{pr}_{j} \circ f$. Let $p \in L \cap u_{\varepsilon}^{-1}\left(L^{\prime}\right)$. Then in a neighborhood (depending on $\varepsilon$ ) of $p$ we have $\psi_{j} \circ u_{\varepsilon}=f_{j} \circ u_{\varepsilon}$, so

$$
D^{(k)}\left(\psi_{j} \circ u_{\varepsilon} \circ \varphi^{-1}\right)(\varphi(p))=D^{(k)}\left(f_{j} \circ u_{\varepsilon} \circ \varphi^{-1}\right)(\varphi(p)),
$$

from which the claim follows.

$(\mathrm{a}) \Rightarrow(\mathrm{c})$ : We have to deduce (c) from Definition 2.1. Let $f \in \mathcal{C}^{\infty}(Y)$ and $K \subset \subset X$. We may assume without loss of generality that $K \subset \subset V$ for some chart $(V, \varphi)$ in $X$. Since $\left(u_{\varepsilon}\right)_{\varepsilon}$ is c-bounded we may choose $K^{\prime} \subset \subset Y$ and $\varepsilon_{0}>0$ such that $u_{\varepsilon}(K) \subseteq K^{\prime}$ for all $\varepsilon<\varepsilon_{0}$. Cover $K^{\prime}$ by charts $\left(W_{l}, \psi_{l}\right)$ in $Y$ $(1 \leq l \leq s)$ and write $K^{\prime}=\bigcup_{l=1}^{s} K_{l}^{\prime}$ with $K_{l}^{\prime} \subset \subset W_{l}$ for each $l$. Let $p \in K$ and choose $l \in\{1, \ldots, s\}$ such that $u_{\varepsilon}(p) \in K_{l}^{\prime}$. Then $f \circ u_{\varepsilon}=\left(f \circ \psi_{l}^{-1}\right) \circ\left(\psi_{l} \circ u_{\varepsilon}\right)$ in a neighborhood of $p$ (depending on $\varepsilon$ ). Applying Definition 2.1 (ii) to $L:=K$, $(V, \varphi), L^{\prime}:=K_{l}^{\prime},\left(W_{l}, \psi_{l}\right)$ we obtain $\varepsilon_{1}=\min _{1 \leq l \leq s} \varepsilon_{1}^{l}, N=\max _{1 \leq l \leq s} N_{l}$, and $C=\max _{1 \leq l \leq s} C_{l}$ such that

$$
\sup _{p \in K}\left\|D^{(k)}\left(f \circ u_{\varepsilon} \circ \varphi^{-1}\right)(\varphi(p))\right\| \leq C \varepsilon^{-N}
$$

for $\varepsilon<\varepsilon_{1}$, so indeed $\left(f \circ u_{\varepsilon}\right)_{\varepsilon} \in \mathcal{E}_{M}(X)$.

(c) $\Rightarrow(\mathrm{b})$ is immediate from Proposition 3.1.

A similar characterization can be derived for the equivalence relation $\sim$ on $\mathcal{E}_{M}[X, Y]$ (see (c), (d) in Theorem 3.3 below). Moreover, this characterization provides an affirmative answer to the question raised in [15], Remark 2.11 whether a characterization of equivalence of elements of spaces of Colombeau generalized functions which does not resort to derivatives of representatives (as established for practically all (scalar) variants of the construction in [11, Th. 13.1) was also attainable in the context of manifold-valued generalized functions.

3.3 Theorem. Let $\left(u_{\varepsilon}\right)_{\varepsilon},\left(v_{\varepsilon}\right)_{\varepsilon} \in \mathcal{E}_{M}[X, Y]$. The following statements are equivalent:

(i) $\left(u_{\varepsilon}\right)_{\varepsilon} \sim\left(v_{\varepsilon}\right)_{\varepsilon}$.

(ii) $\left(u_{\varepsilon}\right)_{\varepsilon} \sim_{0}\left(v_{\varepsilon}\right)_{\varepsilon}$.

(iii) For every Riemannian metric $h$ on $Y$, every $m \in \mathbb{N}$ and every $K \subset \subset X$,

$$
\sup _{p \in K} d_{h}\left(u_{\varepsilon}(p), v_{\varepsilon}(p)\right)=O\left(\varepsilon^{m}\right) \quad(\varepsilon \rightarrow 0) .
$$

(iv) $\left(f \circ u_{\varepsilon}-f \circ v_{\varepsilon}\right)_{\varepsilon} \in \mathcal{N}(X)$ for all $f \in \mathcal{D}(Y)$.

(v) $\left(f \circ u_{\varepsilon}-f \circ v_{\varepsilon}\right)_{\varepsilon} \in \mathcal{N}(X)$ for all $f \in \mathcal{C}^{\infty}(Y)$. 
Proof. Clearly, (i) $\Rightarrow$ (ii).

(ii) $\Leftrightarrow($ iii): see 15], Th. 2.10.

(ii) $\Rightarrow$ (iv): Let $K \subset \subset X, f \in \mathcal{D}(Y)$. By [18], (3) it suffices to show that

$$
\sup _{p \in K}\left|f \circ u_{\varepsilon}(p)-f \circ v_{\varepsilon}(p)\right|=O\left(\varepsilon^{m}\right)
$$

for each $m \in \mathbb{N}$. To see this, choose $K^{\prime} \subset \subset Y$ and $\varepsilon_{0}>0$ such that $u_{\varepsilon}(K) \cup$ $v_{\varepsilon}(K) \subseteq K^{\prime}$ for $\varepsilon<\varepsilon_{0}$. Cover $K^{\prime}$ by open sets $W_{l}^{\prime}$, $\overline{W_{l}^{\prime}} \subset \subset W_{l}$ for charts $\left(W_{l}, \psi_{l}\right)(1 \leq l \leq s)$. From (ii) it follows that for each fixed sufficiently small $\varepsilon$ and each $p \in K$ there exists $l \in\{1, \ldots, s\}$ such that both $u_{\varepsilon}(p)$ and $v_{\varepsilon}(p)$ are contained in $W_{l}^{\prime}$. In this case we have

$$
\begin{aligned}
\left|\left(f \circ u_{\varepsilon}-f \circ v_{\varepsilon}\right)(p)\right| & =\left|\left(\left(f \circ \psi_{l}^{-1}\right) \circ\left(\psi_{l} \circ u_{\varepsilon}\right)-\left(f \circ \psi_{l}^{-1}\right) \circ\left(\psi_{l} \circ v_{\varepsilon}\right)\right)(p)\right| \\
& \leq C\left|\left(\psi_{l} \circ v_{\varepsilon}-\psi_{l} \circ u_{\varepsilon}\right)(p)\right|
\end{aligned}
$$

by 15], Lemma 2.5, where $C$ is independent of $\varepsilon$. Hence the claim follows from Definition 2.3

(iv) $\Rightarrow$ (i): We first have to show that for every Riemannian metric $h$ on $Y$ and any $K \subset \subset X$ we have $\sup _{p \in K} d_{h}\left(u_{\varepsilon}(p), v_{\varepsilon}(p)\right) \rightarrow 0$ as $\varepsilon \rightarrow 0$. Suppose to the contrary that

$$
\exists K \subset \subset X \exists \delta>0 \forall k \in \mathbb{N} \exists \varepsilon_{k}<\frac{1}{k} \exists p_{k} \in K: \quad d_{h}\left(u_{\varepsilon_{k}}\left(p_{k}\right), v_{\varepsilon_{k}}\left(p_{k}\right)\right) \geq \delta .
$$

Since $\left(u_{\varepsilon}\right)_{\varepsilon}$ and $\left(v_{\varepsilon}\right)_{\varepsilon}$ are c-bounded, there exists $K^{\prime} \subset \subset Y$ such that $u_{\varepsilon}(K) \cup$ $v_{\varepsilon}(K) \subseteq K^{\prime}$ for $\varepsilon$ small. Thus, without loss of generality we may suppose that $u_{\varepsilon_{k}}\left(p_{k}\right) \rightarrow q_{1}$ and $v_{\varepsilon_{k}}\left(p_{k}\right) \rightarrow q_{2}$ with $q_{i} \in K^{\prime}$ for $i=1,2$. By (3), $q_{1} \neq q_{2}$. Choose $f \in \mathcal{D}(Y)$ with $f\left(q_{1}\right)=1, f\left(q_{2}\right)=0$. But then

$$
1=\lim _{k \rightarrow \infty}\left|f\left(u_{\varepsilon_{k}}\left(p_{k}\right)\right)-f\left(v_{\varepsilon_{k}}\left(p_{k}\right)\right)\right| \leq \sup _{p \in K}\left|f\left(u_{\varepsilon_{k}}(p)\right)-f\left(v_{\varepsilon_{k}}(p)\right)\right|=O\left(\varepsilon_{k}^{m}\right)
$$

for all $m$, a contradiction.

Finally, we have to establish property (ii) of Definition 2.2. Thus let $L \subset \subset V$, $(V, \varphi)$ a chart in $X, L^{\prime} \subset \subset W,(W, \psi)$ a chart in $Y$. Fix $j \in\{1, \ldots, m\}$ and choose $f_{j} \in \mathcal{D}(W)$ such that $f_{j} \equiv \psi_{j}$ in a neighborhood of $L^{\prime}$. Then for any $p \in L \cap u_{\varepsilon}^{-1}\left(L^{\prime}\right) \cap v_{\varepsilon}^{-1}\left(L^{\prime}\right)$ there exists a neighborhood (depending on $\varepsilon$ ) on which $\psi_{j} \circ u_{\varepsilon}=f_{j} \circ u_{\varepsilon}$ and $\psi_{j} \circ v_{\varepsilon}=f_{j} \circ v_{\varepsilon}$. Hence, since $\left(f_{j} \circ u_{\varepsilon}-f_{j} \circ v_{\varepsilon}\right)_{\varepsilon} \in \mathcal{N}(X)$, for each $k$ in $\mathbb{N}$ we have

$$
\begin{aligned}
& \left\|D^{(k)}\left(\psi_{j} \circ u_{\varepsilon} \circ \varphi^{-1}-\psi_{j} \circ v_{\varepsilon} \circ \varphi^{-1}\right)(\varphi(p))\right\| \\
& \quad \leq \sup _{p^{\prime} \in L}\left\|D^{(k)}\left(f_{j} \circ u_{\varepsilon} \circ \varphi^{-1}-f_{j} \circ v_{\varepsilon} \circ \varphi^{-1}\right)\left(\varphi\left(p^{\prime}\right)\right)\right\|=O\left(\varepsilon^{m}\right)
\end{aligned}
$$

for each $m$, yielding the claim.

(iv) $\Leftrightarrow(\mathrm{v})$ follows as in Proposition 3.2

The above characterization result has several important consequences. First, we obtain a characterization of equivalence of compactly supported generalized points. 
3.4 Corollary. Let $\left(p_{\varepsilon}\right)_{\varepsilon},\left(q_{\varepsilon}\right)_{\varepsilon} \in X^{I}$ be compactly supported. The following statements are equivalent:

(i) $\left(p_{\varepsilon}\right)_{\varepsilon} \sim\left(q_{\varepsilon}\right)_{\varepsilon}$.

(ii) $\left(f\left(p_{\varepsilon}\right)\right)_{\varepsilon} \sim\left(f\left(q_{\varepsilon}\right)\right)_{\varepsilon}$ for all $f \in \mathcal{D}(X)$ (respectively all $f \in \mathcal{C}^{\infty}(X)$ ).

(iii) $\left|f\left(p_{\varepsilon}\right)-f\left(q_{\varepsilon}\right)\right|=O\left(\varepsilon^{m}\right)$ as $\varepsilon \rightarrow 0$ for all $m \in \mathbb{N}$ and all $f \in \mathcal{D}(X)$ (respectively all $f \in \mathcal{C}^{\infty}(X)$ ).

Proof. (i) $\Leftrightarrow\left(\right.$ iii): Setting $u_{\varepsilon}(p) \equiv p_{\varepsilon}$ and $v_{\varepsilon}(p) \equiv q_{\varepsilon}$, the nets $\left(u_{\varepsilon}\right)_{\varepsilon},\left(v_{\varepsilon}\right)_{\varepsilon}$ are contained in $\mathcal{E}_{M}[X, X]$. It is clear from the definitions that $\left(u_{\varepsilon}\right)_{\varepsilon} \sim\left(v_{\varepsilon}\right)_{\varepsilon}$ in $\mathcal{E}_{M}[X, X]$ if and only if $\left(p_{\varepsilon}\right)_{\varepsilon} \sim\left(q_{\varepsilon}\right)_{\varepsilon}$. Thus an application of Theorem 3.3, (i) $\Leftrightarrow$ (iv) gives the result.

(ii) $\Leftrightarrow$ (iii) is immediate from the definition of equivalence of generalized numbers.

Based on Theorem 3.3, the following result provides a point value characterization of elements of $\mathcal{G}[X, Y]$.

3.5 Theorem. Let $u, v \in \mathcal{G}[X, Y]$. Then $u=v$ if and only if $u(\tilde{p})=v(\tilde{p})$ for all $\tilde{p} \in \widetilde{X}_{c}$.

Proof. Immediate from $(\mathrm{a}) \Leftrightarrow(\mathrm{b})$ in Theorem 3.3 and 15 , Prop. 2.14.

Moreover, Theorem 3.3 can be used to show that composition of manifoldvalued generalized functions can be carried out unrestrictedly (i.e., without any additional assumptions, as, e.g., condition (6) in 15).

3.6 Theorem. Let $u=\left[\left(u_{\varepsilon}\right)_{\varepsilon}\right] \in \mathcal{G}[X, Y], v=\left[\left(v_{\varepsilon}\right)_{\varepsilon}\right] \in \mathcal{G}[Y, Z]$. Then $v \circ u:=$ $\left[\left(v_{\varepsilon} \circ u_{\varepsilon}\right)_{\varepsilon}\right]$ is a well-defined element of $\mathcal{G}[X, Z]$.

Proof. The proof of [15], Th. 2.16 shows that $\left(v_{\varepsilon} \circ u_{\varepsilon}\right)_{\varepsilon} \in \mathcal{E}_{M}[X, Y]$. Moreover, it also establishes that $\left(u_{\varepsilon}\right)_{\varepsilon} \sim\left(u_{\varepsilon}^{\prime}\right)_{\varepsilon}$ implies $\left(v_{\varepsilon} \circ u_{\varepsilon}\right) \sim_{0}\left(v_{\varepsilon} \circ u_{\varepsilon}^{\prime}\right)$ and that $\left(v_{\varepsilon}\right)_{\varepsilon} \sim\left(v_{\varepsilon}^{\prime}\right)_{\varepsilon}$ implies $\left(v_{\varepsilon} \circ u_{\varepsilon}\right) \sim_{0}\left(v_{\varepsilon}^{\prime} \circ u_{\varepsilon}\right)$. Hence by Theorem 3.3, the class of $\left(v_{\varepsilon} \circ u_{\varepsilon}\right)_{\varepsilon}$ is well-defined.

\section{Characterization of generalized vector bundle homomorphisms}

The aim of this section is to derive characterizations similar to Proposition 3.2 and Theorem 3.3 also for generalized vector bundle homomorphisms. In the following considerations for any vector bundle $E$ over $X$ the role of the "model spaces" $\mathcal{E}_{M}(X)$ respectively $\mathcal{N}(X)$ (cf. Proposition 3.2 (b), (c) and Theorem 3.3 (iv), (v)) will be played by $\mathcal{E}_{M}^{V B}\left[E, \mathbb{R} \times \mathbb{R}^{m^{\prime}}\right]$ respectively $\sim_{v b}$ on that space. 
In order to allow a smooth presentation of the following results let us therefore examine the simplifications that ensue from the particularly simple structure of the range space $\mathbb{R} \times \mathbb{R}^{m^{\prime}}$. Let $\left(u_{\varepsilon}\right)_{\varepsilon} \in \operatorname{Hom}\left(E, \mathbb{R} \times \mathbb{R}^{m^{\prime}}\right)$ and let $(V, \Phi)$ be a vector bundle chart in $E$. Then using the trivial vector bundle chart id on $\mathbb{R} \times \mathbb{R}^{m^{\prime}}$ we may write

$$
u_{\varepsilon} \circ \Phi^{-1}(x, \xi)=\left(u_{\varepsilon \operatorname{id} \Phi}^{(1)}(x), u_{\varepsilon \operatorname{id} \Phi}^{(2)}(x) \cdot \xi\right) .
$$

It follows that $\left(u_{\varepsilon}\right)_{\varepsilon} \in \mathcal{E}_{M}^{V B}\left[E, \mathbb{R} \times \mathbb{R}^{m^{\prime}}\right]$ if and only if $\left(\underline{u_{\varepsilon}}\right)_{\varepsilon}$ is c-bounded and for each vector bundle chart $(V, \Phi), u_{\varepsilon_{\text {id }} \Phi}^{(1)} \in \mathcal{E}_{M}(\varphi(V))$ and $u_{\varepsilon_{\text {id }} \Phi}^{(2)} \in \mathcal{E}_{M}(\varphi(V))^{\left(m^{\prime}\right)^{2}}$. Moreover, it follows that for $\left(u_{\varepsilon}\right)_{\varepsilon},\left(v_{\varepsilon}\right)_{\varepsilon} \in \mathcal{E}_{M}^{V B}\left[E, \mathbb{R} \times \mathbb{R}^{m^{\prime}}\right],\left(u_{\varepsilon}\right)_{\varepsilon} \sim_{v b}\left(v_{\varepsilon}\right)_{\varepsilon}$ is equivalent to $u_{\varepsilon_{\text {id }} \Phi}^{(1)}-v_{\varepsilon_{\text {id }} \Phi}^{(1)} \in \mathcal{N}(\varphi(V))$ and $u_{\varepsilon_{\text {id }} \Phi}^{(2)}-v_{\varepsilon_{\text {id }} \Phi}^{(2)} \in \mathcal{N}(\varphi(V))$ for each vector bundle chart $(V, \Phi)$. By 11 , Th. 13.1 and the remark following it, this in turn is equivalent to $\left(u_{\varepsilon}\right)_{\varepsilon} \sim_{v b 0}\left(v_{\varepsilon}\right)_{\varepsilon}$, so $\sim_{v b}$ and $\sim_{v b 0}$ are the same for the model space $\mathcal{E}_{M}^{V B}\left[E, \mathbb{R} \times \mathbb{R}^{m^{\prime}}\right]$. We will extend the validity of this observation to general $\mathcal{E}_{M}[E, F]$ in Theorem 4.2 below.

For a vector bundle $E \rightarrow X$ we denote by $\operatorname{Hom}_{c}\left(E, \mathbb{R} \times \mathbb{R}^{m^{\prime}}\right)$ the set of all vector bundle homomorphisms $f$ such that $\underline{f}: X \rightarrow \mathbb{R}$ has compact support.

4.1 Proposition. Let $\left(u_{\varepsilon}\right)_{\varepsilon} \in \operatorname{Hom}(E, F)^{I}$. The following statements are equivalent:

(a) $\left(u_{\varepsilon}\right)_{\varepsilon} \in \mathcal{E}_{M}^{V B}[E, F]$.

(b) (i) $\left(\underline{u_{\varepsilon}}\right)_{\varepsilon}$ is c-bounded.

(ii) $\left(\hat{f} \circ u_{\varepsilon}\right)_{\varepsilon} \in \mathcal{E}_{M}^{V B}\left(E, \mathbb{R} \times \mathbb{R}^{m^{\prime}}\right)$ for all $\hat{f} \in \operatorname{Hom}_{c}\left(F, \mathbb{R} \times \mathbb{R}^{m^{\prime}}\right)$.

(c) $\left(\hat{f} \circ u_{\varepsilon}\right)_{\varepsilon} \in \mathcal{E}_{M}^{V B}\left(E, \mathbb{R} \times \mathbb{R}^{m^{\prime}}\right)$ for all $\hat{f} \in \operatorname{Hom}\left(F, \mathbb{R} \times \mathbb{R}^{m^{\prime}}\right)$.

Proof. $(\mathrm{a}) \Rightarrow(\mathrm{c})$ : By (乘) we have to show that for each $\hat{f} \in \operatorname{Hom}\left(F, \mathbb{R} \times \mathbb{R}^{m^{\prime}}\right)$ and each vector bundle chart $(V, \Phi)$ of $E$ we have $\left(\left(\hat{f} \circ u_{\varepsilon}\right)_{\mathrm{id} \Phi}^{(1)}\right)_{\varepsilon} \in \mathcal{E}_{M}(\varphi(V))$ and $\left(\left(\hat{f} \circ u_{\varepsilon}\right)_{\mathrm{id} \Phi}^{(2)}\right)_{\varepsilon} \in \mathcal{E}_{M}(\varphi(V))^{\left(m^{\prime}\right)^{2}}$. Due to $\left(\hat{f} \circ u_{\varepsilon}\right)_{\mathrm{id} \Phi}^{(1)}=\hat{f} \circ \underline{u}_{\varepsilon} \circ \varphi^{-1}$, the first of these claims follows from Proposition 3.2 since $\left(\underline{u_{\varepsilon}}\right)_{\varepsilon} \in \overline{\mathcal{E}_{M}}[X, Y]$ and $\hat{f} \in \mathcal{C}^{\infty}(Y)$. Concerning the second one, let $L \subset \subset V$ and choose $\varepsilon_{0}>0$, $\bar{L}^{\prime} \subset \subset Y$ such that $u_{\varepsilon}(L) \subseteq L^{\prime}$ for $\varepsilon<\varepsilon_{0}$. Cover $L^{\prime}$ by vector bundle charts $\left(W_{l}, \Psi_{l}\right)$ of $F(1 \leq \bar{l} \leq s)$ and write $L=\bigcup_{l=1}^{s} L_{l}^{\prime}$ with $L_{l}^{\prime} \subset \subset W_{l}$. Choose $\varepsilon_{1}<\varepsilon_{0}$ such that property (ii) of Definition 2.4 is satisfied for $(L, V, \Phi)$ and $\left(L_{1}^{\prime}, W_{1}, \Psi_{1}\right), \ldots,\left(L_{s}^{\prime}, W_{s}, \Psi_{s}\right)$ simultaneously. Let $\varepsilon<\varepsilon_{1}$ and $p \in L$ with $u_{\varepsilon}(p) \in L_{l}^{\prime}$. Then for $p^{\prime}$ in a neighborhood (depending on $\varepsilon$ ) of $p$ we have

$$
\left(\hat{f} \circ u_{\varepsilon}\right)_{\mathrm{id} \Phi}^{(2)}\left(\varphi\left(p^{\prime}\right)\right)=\left(\hat{f} \circ \Psi_{l}^{-1}\right)^{(2)}\left(\psi_{l} \circ \underline{u_{\varepsilon}}\left(p^{\prime}\right)\right) \cdot u_{\varepsilon \Psi_{l} \Phi}^{(2)}\left(\varphi\left(p^{\prime}\right)\right),
$$

so the claim follows.

(c) $\Rightarrow(\mathrm{b})$ : (i) follows from Proposition 3.1 while (ii) is immediate.

$(\mathrm{b}) \Rightarrow(\mathrm{a})$ We have to establish properties (i) and (ii) of Definition 2.4. Since $\left(\hat{f} \circ u_{\varepsilon}\right)_{\mathrm{id} \Phi}^{(1)}=\underline{\hat{f}} \circ \underline{u_{\varepsilon}} \circ \varphi^{-1}$ for any $\hat{f} \in \operatorname{Hom}\left(F, \mathbb{R} \times \mathbb{R}^{m^{\prime}}\right)$, (i) follows from 
Proposition 3.2. Let $L \subset \subset V,(V, \Phi)$ a vector bundle chart in $E, L^{\prime} \subset \subset W$, $(W, \Psi)$ a vector bundle chart in $F$ and choose an open neighborhood $U$ of $L^{\prime}$ whose compact closure is contained in $W$. Choose any $l \in\{1, \ldots, m\}$ and let $\hat{f}_{l} \in \operatorname{Hom}_{c}\left(F, \mathbb{R} \times \mathbb{R}^{m^{\prime}}\right)$ such that

$$
\left.\hat{f}_{l}\right|_{\pi_{Y}^{-1}(U)}=\left.\left(\operatorname{pr}_{l} \times \mathrm{id}_{\mathbb{R}^{m^{\prime}}}\right) \circ \Psi\right|_{\pi_{Y}^{-1}(U)}
$$

where $\operatorname{pr}_{l}: \mathbb{R}^{m} \rightarrow \mathbb{R}$. Let $p \in L \cap \underline{u}_{\varepsilon}^{-1}\left(L^{\prime}\right)$. Then for $p^{\prime}$ in a suitable neighborhood of $p$ we have

$$
\left(\hat{f}_{l} \circ u_{\varepsilon} \circ \Phi^{-1}\right)^{(2)}\left(\varphi\left(p^{\prime}\right)\right)=u_{\varepsilon \Psi \Phi}^{(2)}\left(\varphi\left(p^{\prime}\right)\right),
$$

so the desired estimates of $D^{(k)}\left(u_{\varepsilon \Psi \Phi}^{(2)}\right)(\varphi(p))$ follow, thereby establishing property (ii) of Definition 2.4.

We now characterize the equivalence relation $\sim_{v b}$ on $\mathcal{E}_{M}^{V B}[E, F]$, simultaneously establishing that vb-equivalence is in fact identical to vb-0-equivalence.

4.2 Theorem. Let $\left(u_{\varepsilon}\right)_{\varepsilon},\left(v_{\varepsilon}\right)_{\varepsilon} \in \mathcal{E}_{M}^{V B}(E, F)$. The following statements are equivalent:

(i) $\left(u_{\varepsilon}\right)_{\varepsilon} \sim_{v b}\left(v_{\varepsilon}\right)_{\varepsilon}$.

(ii) $\left(u_{\varepsilon}\right)_{\varepsilon} \sim_{v b 0}\left(v_{\varepsilon}\right)_{\varepsilon}$.

(iii) $\left(\hat{f} \circ u_{\varepsilon}\right) \sim_{v b}\left(\hat{f} \circ v_{\varepsilon}\right)_{\varepsilon}$ in $\mathcal{E}_{M}^{V B}\left[E, \mathbb{R} \times \mathbb{R}^{m^{\prime}}\right]$ for all $\hat{f} \in \operatorname{Hom}_{c}\left(E, \mathbb{R} \times \mathbb{R}^{m^{\prime}}\right)$.

(iv) $\left(\hat{f} \circ u_{\varepsilon}\right) \sim_{v b}\left(\hat{f} \circ v_{\varepsilon}\right)_{\varepsilon}$ in $\mathcal{E}_{M}^{V B}\left[E, \mathbb{R} \times \mathbb{R}^{m^{\prime}}\right]$ for all $\hat{f} \in \operatorname{Hom}\left(E, \mathbb{R} \times \mathbb{R}^{m^{\prime}}\right)$.

Proof. (i) $\Rightarrow$ (ii) is clear.

(ii) $\Rightarrow$ (iv): Let $\hat{f} \in \operatorname{Hom}\left(E, \mathbb{R} \times \mathbb{R}^{m^{\prime}}\right)$ and $L \subset \subset V,(V, \Phi)$ a vector bundle chart in $E$. To prove (iv), by the remarks following (何) it suffices to establish the $\mathcal{N}$-estimates of order 0 on $L$ for both $\left(\hat{f} \circ u_{\varepsilon}-\hat{f} \circ v_{\varepsilon}\right)_{\mathrm{id} \Phi}^{(1)}$ and $\left(\hat{f} \circ u_{\varepsilon}-\hat{f} \circ v_{\varepsilon}\right)_{\mathrm{id} \Phi}^{(2)}$. Choose $K^{\prime} \subset \subset Y$ and $\varepsilon_{1}>0$ such that $\underline{u_{\varepsilon}}(L) \cup \underline{v_{\varepsilon}}(L) \subseteq K^{\prime}$ for $\varepsilon<\varepsilon_{1}$. Cover $K^{\prime}$ by open sets $W_{l}^{\prime}, \overline{W_{l}^{\prime}} \subset \subset W_{l}(1 \leq l \leq s)$, where $\left.\overline{\left(W_{l}\right.}, \Psi_{l}\right)$ are vector bundle charts in $F$. Since for any Riemannian metric $h$ on $Y, \sup _{p \in K} d_{h}\left(\underline{u_{\varepsilon}}(p), \underline{v_{\varepsilon}}(p)\right) \rightarrow 0$ as $\varepsilon \rightarrow 0$, for each $\varepsilon$ small and each $p \in K$ there exists $l \in\{1, \ldots, s\}$ such that both $\underline{u_{\varepsilon}}(p)$ and $\underline{v_{\varepsilon}}(p)$ are contained in $W_{l}^{\prime}$. Now the estimate for

$$
\left(\hat{f} \circ u_{\varepsilon}-\hat{f} \circ v_{\varepsilon}\right)_{\mathrm{id} \Phi}^{(1)}(\varphi(p))=\left(\underline{\hat{f}} \circ \underline{u_{\varepsilon}}-\underline{\hat{f}} \circ \underline{v_{\varepsilon}}\right) \circ \varphi^{-1}(p)
$$

follows from Theorem 3.3 (ii) $\Rightarrow(\mathrm{v})$, while that for $\left(\hat{f} \circ u_{\varepsilon}-\hat{f} \circ v_{\varepsilon}\right)_{\mathrm{id} \Phi}^{(2)}(\varphi(p))$ is derived from a representation as in (5) using Definition 2.5 (ii) (for $k=0$ ) and Lemma 2.5 of 15]: again the constants appearing due to the application of that result can be chosen independently of $\varepsilon$ since the domain of $\left(\hat{f} \circ \Psi_{l}^{-1}\right)^{(2)}$ is an open neighborhood of $\psi_{l}\left(\overline{W_{l}^{\prime}}\right)$. 
(iv) $\Rightarrow$ (iii): Clear.

(iii) $\Rightarrow$ (i) Using (7), property (i) of Definition 2.5 follows from Theorem 3.3 (v) $\Rightarrow$ (i). Finally, property (ii) of that definition is established by employing an $\hat{f}_{l}$ as in the proof of Proposition 4.1, (b) $\Rightarrow$ (a), using a representation of both $u_{\varepsilon \Psi \Phi}^{(2)}$ and $v_{\varepsilon \Psi \Phi}^{(2)}$ as in (6).

The above result can in turn be used to derive the following characterization result for generalized vector bundle points.

4.3 Corollary. Let $\left(e_{\varepsilon}\right)_{\varepsilon},\left(e_{\varepsilon}^{\prime}\right)_{\varepsilon} \in E^{I}$ be vb-moderate. The following statements are equivalent:

(i) $\left(e_{\varepsilon}\right)_{\varepsilon} \sim_{v b}\left(e_{\varepsilon}^{\prime}\right)_{\varepsilon}$.

(ii) $\left(\hat{f}\left(e_{\varepsilon}\right)\right)_{\varepsilon} \sim_{v b}\left(\hat{f}\left(e_{\varepsilon}^{\prime}\right)\right)_{\varepsilon}$ in $\mathbb{R} \times \mathbb{R}^{n^{\prime}}$ for each $\hat{f} \in \operatorname{Hom}_{c}\left(E, \mathbb{R} \times \mathbb{R}^{n^{\prime}}\right)$ (respectively $\left.\in \operatorname{Hom}\left(E, \mathbb{R} \times \mathbb{R}^{n^{\prime}}\right)\right)$.

(iii) $\left|\hat{f}\left(e_{\varepsilon}\right)-\hat{f}\left(e_{\varepsilon}^{\prime}\right)\right|=O\left(\varepsilon^{m}\right)$ as $\varepsilon \rightarrow 0$ for all $m \in \mathbb{N}$ and all $\hat{f} \in \operatorname{Hom}_{c}(E, \mathbb{R} \times$ $\left.\mathbb{R}^{n^{\prime}}\right)$ (respectively $\in \operatorname{Hom}\left(E, \mathbb{R} \times \mathbb{R}^{n^{\prime}}\right)$ ).

Proof. (i) $\Leftrightarrow$ (ii) follows from applying Theorem 4.2 to $u_{\varepsilon} \equiv e_{\varepsilon}, v_{\varepsilon} \equiv e_{\varepsilon}^{\prime}$. (ii) $\Leftrightarrow$ (iii) is immediate from the definitions.

We can employ this result in order to get rid of the additional technical assumption in the statement of [15], Prop. 3.6 (ii) (called (6) there). Hence generalized vector bundle points may be inserted into generalized vector bundle homomorphisms unrestrictedly.

4.4 Corollary. Let $u=\left[\left(u_{\varepsilon}\right)_{\varepsilon}\right] \in \operatorname{Hom}_{\mathcal{G}}[E, F]$, $\tilde{e}=\left[\left(e_{\varepsilon}\right)_{\varepsilon}\right] \in E_{c}^{\sim v b}$. Then $u(\tilde{e}):=\left[\left(u_{\varepsilon}\left(e_{\varepsilon}\right)\right)_{\varepsilon}\right]$ is a well-defined element of of $F_{c}^{\sim v b}$.

Proof. The proof of [15], Prop. 3.6 (ii) shows (even under the more general assumptions made here) that $\left(u_{\varepsilon}\left(e_{\varepsilon}\right)\right)_{\varepsilon}$ is vb-moderate and that $\left(u_{\varepsilon}\right)_{\varepsilon} \sim_{v b}\left(u_{\varepsilon}^{\prime}\right)_{\varepsilon}$ implies $\left(u_{\varepsilon}\left(e_{\varepsilon}\right)\right)_{\varepsilon} \sim_{v b}\left(u_{\varepsilon}^{\prime}\left(e_{\varepsilon}\right)\right)_{\varepsilon}$ for any vb-moderate $\left(e_{\varepsilon}\right)_{\varepsilon}$. To finish the proof it remains to show that $\left(e_{\varepsilon}\right)_{\varepsilon} \sim\left(e_{\varepsilon}^{\prime}\right)_{\varepsilon}$ implies $\left(u_{\varepsilon}\left(e_{\varepsilon}\right)\right)_{\varepsilon} \sim_{v b}\left(u_{\varepsilon}\left(e_{\varepsilon}^{\prime}\right)\right)_{\varepsilon}$ even for general $\left(u_{\varepsilon}\right)_{\varepsilon} \in \mathcal{E}_{M}^{V B}[E, F]$. Let $\hat{f} \in \operatorname{Hom}_{c}\left(F, \mathbb{R} \times \mathbb{R}^{m^{\prime}}\right)$. By Corollary 4.3 (ii) it suffices to establish $\left(\left(\hat{f} \circ u_{\varepsilon}\right)\left(e_{\varepsilon}\right)\right)_{\varepsilon} \sim_{v b}\left(\left(\hat{f} \circ u_{\varepsilon}\right)\left(e_{\varepsilon}^{\prime}\right)\right)_{\varepsilon}$. Now $\left(\hat{f} \circ u_{\varepsilon}\right)_{\varepsilon} \in$ $\mathcal{E}_{M}^{V B}\left[E, \mathbb{R} \times \mathbb{R}^{m^{\prime}}\right]$ by Proposition 4.1 and by Ex. 2.15 (i) of [15], $\left(\hat{f} \circ u_{\varepsilon}\right)_{\varepsilon}$ satisfies (6) from [15]. Thus the claim follows from [15], Prop. 3.6 (ii).

The above result enables us to conclude the following point value characterization of generalized vector bundle homomorphisms.

4.5 Theorem. Let $u, v \in \operatorname{Hom}_{\mathcal{G}}[E, F]$. Then $u=v$ if and only if $u(\tilde{e})=v(\tilde{e})$ in $F_{c}^{\sim}{ }_{v b}$ for all $\tilde{e} \in E_{c}^{\sim v b}$. 
Proof. By Corollary 4.4, the point values of $u$ and $v$ at $\tilde{e}$ are well-defined. In particular, $u=v$ entails $u(\tilde{e})=v(\tilde{e})$ for all $\tilde{e} \in E_{c}^{\sim^{v b}}$. The converse direction follows from the proof of 15, Th. 3.7 (ii) and an application of Theorem 4.2, (i) $\Leftrightarrow($ ii).

Furthermore, it now follows that composition of generalized vector bundle homomorphisms can be carried out unrestrictedly:

4.6 Theorem. Let $u=\left[\left(u_{\varepsilon}\right)_{\varepsilon}\right] \in \operatorname{Hom}_{\mathcal{G}}[E, F], v=\left[\left(v_{\varepsilon}\right)_{\varepsilon}\right] \in \operatorname{Hom}_{\mathcal{G}}[F, G]$. Then $v \circ u:=\left[\left(v_{\varepsilon} \circ u_{\varepsilon}\right)_{\varepsilon}\right]$ is a well-defined element of $\operatorname{Hom}_{\mathcal{G}}[E, G]$.

Proof. In order to show that $\left(v_{\varepsilon} \circ u_{\varepsilon}\right)_{\varepsilon} \in \mathcal{E}_{M}^{V B}[E, G]$, by Proposition 4.1 it suffices to show that $\left(\hat{f} \circ v_{\varepsilon} \circ u_{\varepsilon}\right) \in \mathcal{E}_{M}^{V B}\left[E, \mathbb{R} \times \mathbb{R}^{k^{\prime}}\right]$ for each $\hat{f} \in \operatorname{Hom}_{c}\left[G, \mathbb{R} \times \mathbb{R}^{k^{\prime}}\right]$. Moreover, also by Proposition 4.1 we know that for each $\hat{f} \in \operatorname{Hom}_{c}\left[G, \mathbb{R} \times \mathbb{R}^{k^{\prime}}\right]$ $\left(\hat{f} \circ v_{\varepsilon}\right)_{\varepsilon}$ is an element of $\mathcal{E}_{M}^{V B}\left[F, \mathbb{R} \times \mathbb{R}^{k^{\prime}}\right]$. We may therefore assume without loss of generality that $G=\mathbb{R} \times \mathbb{R}^{k^{\prime}} .\left(\underline{v_{\varepsilon}} \circ u_{\varepsilon}\right)_{\varepsilon}=\left(\underline{v_{\varepsilon}} \circ u_{\varepsilon}\right)_{\varepsilon} \in \mathcal{E}_{M}[X, Z]$ by Theorem 3.6. Let $(V, \Phi)$ be any vector bundle chart in $E$. By the remarks following (国) it remains to establish the $\mathcal{E}_{M^{-}}$-estimates for $\left(v_{\varepsilon} \circ u_{\varepsilon}\right)_{\mathrm{id} \Phi}^{(2)} \circ \varphi$ on every $K \subset \subset V$. Choose $\varepsilon_{1}>0$ and $K^{\prime} \subset \subset Y$ such that $\underline{u_{\varepsilon}}(K) \subseteq K^{\prime}$ for all $\varepsilon<\varepsilon_{1}$. Cover $K^{\prime}$ by open sets $W_{1}, \ldots, W_{l}$ for vector bundle charts $\left(W_{i}, \Psi_{i}\right)$ and write $K^{\prime}=\bigcup_{i=1}^{l} K_{i}^{\prime}$ with $K_{i}^{\prime} \subset \subset W_{i}(1 \leq i \leq l)$. Let $p \in K$ and $\varepsilon<\varepsilon_{1}$. Choose $i \in\{1, \ldots, l\}$ such that $\underline{u_{\varepsilon}}(p) \in K_{i}^{\prime}$. Then for $p^{\prime}$ in a neighborhood of $p$ we have

$$
\left(v_{\varepsilon} \circ u_{\varepsilon}\right)_{\operatorname{id} \Phi}^{(2)}\left(\varphi\left(p^{\prime}\right)\right)=v_{\varepsilon \operatorname{id} \Psi_{i}}^{(2)}\left(\psi_{i} \circ \underline{u_{\varepsilon}}\left(p^{\prime}\right)\right) \cdot u_{\varepsilon \Psi_{i} \Phi}^{(2)}\left(\varphi\left(p^{\prime}\right)\right) .
$$

Hence the desired estimates for $D^{(k)}\left(\left(v_{\varepsilon} \circ u_{\varepsilon}\right)_{\mathrm{id} \Phi}^{(2)}\right)(\varphi(p))$ follow from the moderateness-estimates of $v_{\varepsilon \text { id } \Psi_{i}}^{(2)}$ on $\psi_{i}\left(K_{i}^{\prime}\right)$ and property (ii) of Definition 2.4, applied to $u_{\varepsilon \Psi_{i} \Phi}^{(2)}$ and the set of data $(K, V, \Phi),\left(K_{i}^{\prime}, W_{i}, \Psi_{i}\right)$.

To show that $\left[\left(v_{\varepsilon} \circ u_{\varepsilon}\right)_{\varepsilon}\right]$ is well-defined we resort to the point value characterization derived in Theorem 4.6: if $\left(u_{\varepsilon}\right)_{\varepsilon} \sim_{v b}\left(u_{\varepsilon}^{\prime}\right)_{\varepsilon}$ then for any $\tilde{e}=\left[\left(e_{\varepsilon}\right)_{\varepsilon}\right]$ we have $\left(u_{\varepsilon}\left(e_{\varepsilon}\right)\right)_{\varepsilon} \sim_{v b}\left(u_{\varepsilon}^{\prime}\left(e_{\varepsilon}\right)\right)_{\varepsilon}$, hence $\left(v_{\varepsilon} \circ u_{\varepsilon}\left(e_{\varepsilon}\right)\right)_{\varepsilon} \sim_{v b}\left(v_{\varepsilon} \circ u_{\varepsilon}\left(e_{\varepsilon}\right)\right)_{\varepsilon}$ and therefore $\left(v_{\varepsilon} \circ u_{\varepsilon}\right)_{\varepsilon} \sim_{v b}\left(v_{\varepsilon} \circ u_{\varepsilon}\right)_{\varepsilon}$. Similarly, $\left(v_{\varepsilon}\right)_{\varepsilon} \sim_{v b}\left(v_{\varepsilon}^{\prime}\right)_{\varepsilon}$ entails $\left(v_{\varepsilon} \circ u_{\varepsilon}\right)_{\varepsilon} \sim_{v b}\left(v_{\varepsilon} \circ u_{\varepsilon}\right)_{\varepsilon}$, which concludes the proof.

\section{Generalized vector bundle homomorphisms on a generalized mapping}

This section is devoted to an analysis of the hybrid Colombeau spaces introduced in 19], Sec. 4. For the convenience of the reader we recall the basic definitions, namely of the space of moderate nets in $\mathcal{E}^{h}[X, F]=\mathcal{C}^{\infty}(X, F)^{I}$ and the appropriate notion of equivalence in this space. 
5.1 Definition. We define $\mathcal{E}_{M}^{h}[X, F]$ as the set of all nets $\left(u_{\varepsilon}\right)_{\varepsilon} \in \mathcal{E}^{h}[X, F]$ satisfying

(i) $\forall K \subset \subset X \exists K^{\prime} \subset \subset Y \exists \varepsilon_{0}>0 \forall \varepsilon<\varepsilon_{0} \underline{u_{\varepsilon}}(K) \subseteq K^{\prime}$.

(ii) $\forall k \in \mathbb{N}_{0} \forall(V, \varphi)$ chart in $X \forall(W, \Psi)$ vector bundle chart in $F \forall L \subset \subset$ $V \forall L^{\prime} \subset \subset W \exists N \in \mathbb{N} \exists \varepsilon_{1}>0 \exists C>0$ such that

$$
\left\|D^{(k)}\left(\Psi \circ u_{\varepsilon} \circ \varphi^{-1}\right)(\varphi(p))\right\| \leq C \varepsilon^{-N}
$$

for each $\varepsilon<\varepsilon_{1}$ and each $p \in L \cap{\underline{u_{\varepsilon}}}^{-1}\left(L^{\prime}\right)$.

Note that in particular, $\left(u_{\varepsilon}\right)_{\varepsilon} \in \mathcal{E}_{M}^{h}[X, F]$ implies $\left(\underline{u_{\varepsilon}}\right)_{\varepsilon} \in \mathcal{E}_{M}[X, Y]$.

5.2 Definition. $\left(u_{\varepsilon}\right)_{\varepsilon},\left(v_{\varepsilon}\right)_{\varepsilon} \in \mathcal{E}_{M}^{h}[X, F]$ are called equivalent, $\left(u_{\varepsilon}\right)_{\varepsilon} \sim_{h}\left(v_{\varepsilon}\right)_{\varepsilon}$, if the following conditions are satisfied:

(i) For each $K \subset \subset X, \sup _{p \in X} d_{h}\left(\underline{u_{\varepsilon}}, \underline{v_{\varepsilon}}\right) \rightarrow 0$

(ii) $\forall k \in \mathbb{N}_{0} \forall m \in \mathbb{N} \forall(V, \varphi)$ chart in $X, \forall(W, \Psi)$ vector bundle chart in $F$, $\forall L \subset \subset V \forall L^{\prime} \subset \subset W \exists \varepsilon_{1}>0 \exists C>0$ such that

$$
\left\|D^{(k)}\left(\Psi \circ u_{\varepsilon} \circ \varphi^{-1}-\Psi \circ v_{\varepsilon} \circ \varphi^{-1}\right)(\varphi(p))\right\| \leq C \varepsilon^{m}
$$

for each $\varepsilon<\varepsilon_{1}$ and each $p \in L \cap \underline{u}_{\varepsilon}^{-1}\left(L^{\prime}\right) \cap \underline{v}^{-1}\left(L^{\prime}\right)$.

The space of hybrid Colombeau generalized functions from the manifold $X$ into the vector bundle $F$ as usual is defined by $\mathcal{G}^{h}[X, F]:=\mathcal{E}_{M}^{h}[X, F] / \sim_{h}$. We now start by establishing characterizations of moderateness respectively equivalence analogous to the previous cases.

5.3 Proposition. Let $\left(u_{\varepsilon}\right)_{\varepsilon} \in \mathcal{C}^{\infty}(X, F)^{I}$. The following statements are equivalent:

(a) $\left(u_{\varepsilon}\right)_{\varepsilon} \in \mathcal{E}_{M}^{h}[X, F]$.

(b) (i) $\left(\underline{u_{\varepsilon}}\right)_{\varepsilon}$ is c-bounded.

(ii) $\left(\hat{f} \circ u_{\varepsilon}\right)_{\varepsilon} \in \mathcal{E}_{M}^{h}\left[X, \mathbb{R} \times \mathbb{R}^{m^{\prime}}\right]$ for all $\hat{f} \in \operatorname{Hom}_{c}\left(F, \mathbb{R} \times \mathbb{R}^{m^{\prime}}\right)$.

(c) $\left(\hat{f} \circ u_{\varepsilon}\right)_{\varepsilon} \in \mathcal{E}_{M}^{h}\left[X, \mathbb{R} \times \mathbb{R}^{m^{\prime}}\right]$ for all $\hat{f} \in \operatorname{Hom}\left(F, \mathbb{R} \times \mathbb{R}^{m^{\prime}}\right)$.

Proof. (a) $\Rightarrow$ (c): We have to show that for each $\hat{f}=\left(\underline{\hat{f}} \circ \pi_{Y}, \hat{f}_{2}\right) \in \operatorname{Hom}(F, \mathbb{R} \times$ $\mathbb{R}^{m^{\prime}}$ ) (with $\hat{f}_{2}=\operatorname{pr}_{2} \circ \hat{f}, \operatorname{pr}_{2}: \mathbb{R} \times \mathbb{R}^{m^{\prime}} \rightarrow \mathbb{R}^{m^{\prime}}$ ) and each chart $(V, \varphi)$ in $X$ we have $\underline{\hat{f}} \circ \underline{u_{\varepsilon}} \circ \varphi^{-1} \in \mathcal{E}_{M}(\varphi(V))$ and $\left(\hat{f}_{2} \circ u_{\varepsilon} \circ \varphi^{-1}\right)_{\varepsilon} \in \mathcal{E}_{M}(\varphi(V))^{m^{\prime}}$. The first claim follows from Proposition 3.2 since $\left(\underline{u_{\varepsilon}}\right)_{\varepsilon} \in \mathcal{E}_{M}[X, Y]$ and $\hat{f} \in \mathcal{C}^{\infty}(Y)$. Let $L \subset \subset V$ and choose $\varepsilon_{0}>0, L^{\prime} \subset \subset Y$ such that $u_{\varepsilon}(L) \subseteq L^{\prime}$ for $\varepsilon<\varepsilon_{0}$. Choose a covering of $L^{\prime}$ by vector bundle charts $\left(W_{l}, \widehat{\left.\Psi_{l}\right)}\right.$ of $F(1 \leq l \leq s)$ and write $L=\bigcup_{l=1}^{s} L_{l}^{\prime}$ with $L_{l}^{\prime} \subset \subset W_{l}$. There exists $\varepsilon_{1}<\varepsilon_{0}$ such that property (ii) of Definition 5.1 is satisfied for $(L, V, \varphi)$ and $\left(L_{1}^{\prime}, W_{1}, \Psi_{1}\right), \ldots,\left(L_{s}^{\prime}, W_{s}, \Psi_{s}\right)$ 
simultaneously. Let $\varepsilon<\varepsilon_{1}$ and $p \in L$ with $u_{\varepsilon}(p) \in L_{l}^{\prime}$. Then in a neighborhood (depending on $\varepsilon$ ) of $\varphi(p)$ we have

$$
\hat{f}_{2} \circ u_{\varepsilon} \circ \varphi^{-1}=\left(\hat{f}_{2} \circ \Psi_{l}^{-1}\right) \circ\left(\Psi_{l} \circ u_{\varepsilon} \circ \varphi^{-1}\right)
$$

yielding the claim (as in the proof of Proposition $3.2(\mathrm{a}) \Rightarrow(b)$ ).

(c) $\Rightarrow$ (b) follows from Proposition 3.1.

(b) $\Rightarrow$ (a): (i) in Definition 5.1 being evident, let us establish (ii). Let $L \subset \subset(V, \varphi)$ a chart in $X$ and $L^{\prime} \subset \subset(W, \Psi)$ a vector bundle chart in $F$ and choose an open neighborhood $U$ of $L^{\prime}$ whose compact closure is contained in $W$. Choose any $l \in\{1, \ldots, m\}$ and let $\hat{f}_{l} \in \operatorname{Hom}_{c}\left(F, \mathbb{R} \times \mathbb{R}^{m^{\prime}}\right)$ such that

$$
\left.\hat{f}_{l}\right|_{\pi_{Y}^{-1}(U)}=\left.\left(\operatorname{pr}_{l} \times \mathrm{id}_{\mathbb{R}^{m^{\prime}}}\right) \circ \Psi\right|_{\pi_{Y}^{-1}(U)}
$$

where $\operatorname{pr}_{l}: \mathbb{R}^{m} \rightarrow \mathbb{R}$. Let $p \in L \cap \underline{u}^{-1}\left(L^{\prime}\right)$. Then in some neighborhood of $p$ we have

$$
\left(\hat{f}_{l} \circ u_{\varepsilon} \circ \varphi^{-1}\right)=\left(\psi_{l} \circ \underline{u_{\varepsilon}} \circ \varphi^{-1}, \boldsymbol{\psi} \circ u_{\varepsilon} \circ \varphi^{-1}\right)
$$

from which the claim follows.

In the following result, $\sim_{h 0}$ denotes the equivalence relation on $\mathcal{E}_{M}^{h}[X, F]$ defined by imposing Definition 5.2 (i) and (ii) for $k=0$.

5.4 Theorem. Let $\left(u_{\varepsilon}\right)_{\varepsilon},\left(v_{\varepsilon}\right)_{\varepsilon} \in \mathcal{E}_{M}^{h}[X, F]$. The following statements are equivalent:

(i) $\left(u_{\varepsilon}\right)_{\varepsilon} \sim_{h}\left(v_{\varepsilon}\right)_{\varepsilon}$.

(ii) $\left(u_{\varepsilon}\right)_{\varepsilon} \sim_{h 0}\left(v_{\varepsilon}\right)_{\varepsilon}$.

(iii) $\left(\hat{f} \circ u_{\varepsilon}\right) \sim_{h}\left(\hat{f} \circ v_{\varepsilon}\right)_{\varepsilon}$ in $\mathcal{E}_{M}^{h}\left[X, \mathbb{R} \times \mathbb{R}^{m^{\prime}}\right]$ for all $\hat{f} \in \operatorname{Hom}_{c}\left(F, \mathbb{R} \times \mathbb{R}^{m^{\prime}}\right)$.

(iv) $\left(\hat{f} \circ u_{\varepsilon}\right) \sim_{h}\left(\hat{f} \circ v_{\varepsilon}\right)_{\varepsilon}$ in $\mathcal{E}_{M}^{h}\left[X, \mathbb{R} \times \mathbb{R}^{m^{\prime}}\right]$ for all $\hat{f} \in \operatorname{Hom}\left(F, \mathbb{R} \times \mathbb{R}^{m^{\prime}}\right)$.

Proof. (i) $\Rightarrow$ (ii) is clear.

(ii) $\Rightarrow$ (iv): Let $\hat{f} \in \operatorname{Hom}\left(F, \mathbb{R} \times \mathbb{R}^{m^{\prime}}\right)$ and $L \subset \subset V,(V, \varphi)$ a chart in $X$. Using the same notations as in the proof of Proposition 5.3, to show (iv) we have to establish the $\mathcal{N}$-estimates of order 0 on $L$ for $\left(\hat{f} \circ u_{\varepsilon}-\hat{f} \circ v_{\varepsilon}\right)_{\varepsilon}$ and $\left(\hat{f}_{2} \circ u_{\varepsilon}-\hat{f}_{2} \circ v_{\varepsilon}\right)_{\varepsilon}$. Let $L^{\prime} \subset \subset Y$ and $\varepsilon_{0}>0$ such that $\underline{u_{\varepsilon}}(L) \cup \underline{v_{\varepsilon}}(\bar{L}) \subseteq \overline{L^{\prime}}$ for $\varepsilon<\varepsilon_{0}$ and cover $L^{\prime}$ by open sets $W_{l}^{\prime}, \overline{W_{l}^{\prime}} \subset \subset W_{l}(1 \leq l \leq \bar{s})$ with $\left(W_{l}, \Psi_{l}\right)$ vector bundle charts in $F$. Since $\left(\underline{u_{\varepsilon}}\right)_{\varepsilon} \sim_{0}\left(\underline{v_{\varepsilon}}\right)_{\varepsilon}$, for each small $\varepsilon$ and each $p \in L$ there exists $l \in\{1, \ldots, s\}$ such that $\underline{u_{\varepsilon}}(p)$ and $\underline{v_{\varepsilon}}(p)$ are contained in $W_{l}^{\prime}$ simultaneously. Hence the first of the above estimates follows from Theorem 3.3. The second one follows from (8), Definition 5.2 (ii) (for $k=0$ ) and Lemma 2.5 of [15]: the constants in the ensuing estimates can be chosen independently of $\varepsilon$ since the domain of $\hat{f}_{2} \circ \Psi_{l}^{-1}$ is an open neighborhood of $\boldsymbol{\psi}_{l}\left(\overline{W_{l}^{\prime}}\right) \times \mathbb{R}^{m^{\prime}}$.

(iv) $\Rightarrow$ (iii): Obvious. 
(iii) $\Rightarrow$ (i) Property (i) of Definition 5.2 is immediate from Theorem 3.3 . Property (ii) is established using an $\hat{f}_{l}$ as in the proof of Proposition 5.3, (b) $\Rightarrow$ (a), employing representations as in (9) for both $\hat{f}_{l} \circ u_{\varepsilon} \circ \varphi^{-1}$ and $\hat{f}_{l} \circ v_{\varepsilon} \circ \varphi^{-1}$.

As in the previous cases we now use the above results to gain a point value description of hybrid generalized functions.

5.5 Theorem. Let $u=\left[\left(u_{\varepsilon}\right)_{\varepsilon}\right] \in \mathcal{G}^{h}[X, F], \tilde{p}=\left[\left(p_{\varepsilon}\right)_{\varepsilon}\right] \in \widetilde{X}_{c}$. Then

(i) $u(\tilde{p}):=\left[\left(u_{\varepsilon}\left(p_{\varepsilon}\right)\right)_{\varepsilon}\right]$ is a well-defined element of $F_{c}^{\sim v b}$.

(ii) If $u, v \in \mathcal{G}^{h}[X, F]$ then $u=v$ if and only if $u(\tilde{p})=v(\tilde{p})$ for all $\tilde{p} \in \widetilde{X}_{c}$.

Proof. (i) Since $\left(p_{\varepsilon}\right)_{\varepsilon}$ is compactly supported, vb-moderateness of $\left(u_{\varepsilon}\left(p_{\varepsilon}\right)\right)_{\varepsilon}$ follows immediately from c-boundedness of $\left(\underline{u}_{\varepsilon}\right)_{\varepsilon}$ and Definition 5.1. Suppose that $\left(p_{\varepsilon}\right)_{\varepsilon} \sim\left(p_{\varepsilon}^{\prime}\right)_{\varepsilon}$. To show that $\left(u_{\varepsilon}\left(p_{\varepsilon}\right)\right)_{\varepsilon} \sim_{v b}\left(u_{\varepsilon}\left(p_{\varepsilon}^{\prime}\right)\right)_{\varepsilon}$, by Theorem 4.2 and Corollary 4.3 we may without loss of generality suppose that $F=\mathbb{R} \times$ $\mathbb{R}^{m^{\prime}}$. Choose $K \subset \subset X$ such that $p_{\varepsilon}, p_{\varepsilon}^{\prime} \in K$ for $\varepsilon$ small, cover $K$ by charts $\left(V_{1}, \varphi_{1}\right), \ldots,\left(V_{l}, \varphi_{l}\right)$ and write $K=\bigcup_{i=1}^{l} L_{i}$ with $L_{i} \subset \subset V_{i}$. Then choosing the trivial vector bundle chart on $\mathbb{R} \times \mathbb{R}^{m^{\prime}}$ the claim follows from estimates of the form

$$
\left|u_{\varepsilon}\left(p_{\varepsilon}\right)-u_{\varepsilon}\left(p_{\varepsilon}^{\prime}\right)\right| \leq \sup _{q \in L_{i}}\left\|D\left(u_{\varepsilon} \circ \varphi_{i}^{-1}\right)\left(\varphi_{i}(q)\right)\right\|\left|\varphi_{i}\left(p_{\varepsilon}\right)-\varphi_{i}\left(p_{\varepsilon}^{\prime}\right)\right|,
$$

and moderateness of $\left(u_{\varepsilon}\right)_{\varepsilon}$. Finally, let $\left(u_{\varepsilon}\right)_{\varepsilon} \sim_{h}\left(u_{\varepsilon}^{\prime}\right)_{\varepsilon}$. Then $\left(u_{\varepsilon}\left(p_{\varepsilon}\right)\right)_{\varepsilon} \sim_{v b}$ $\left(u_{\varepsilon}^{\prime}\left(p_{\varepsilon}\right)\right)_{\varepsilon}$ follows from Definition 5.2 (ii).

(ii) Necessity has been established in (i). Conversely, suppose that $\left(u_{\varepsilon}\right)_{\varepsilon} \chi_{h}$ $\left(v_{\varepsilon}\right)_{\varepsilon}$, i.e., $\left(u_{\varepsilon}\right)_{\varepsilon} \chi_{h 0}\left(v_{\varepsilon}\right)_{\varepsilon}$ (Theorem 5.4). Then either $\left(\underline{u_{\varepsilon}}\right)_{\varepsilon} \chi_{0}\left(\underline{v_{\varepsilon}}\right)_{\varepsilon}$ in which case by [15], Prop. 2.14 we obtain a $\tilde{p} \in \widetilde{X}_{c}$ with $\underline{u}(\tilde{p}) \neq \underline{v}(\tilde{p})$, and, consequently, $u(\tilde{p}) \neq v(\tilde{p})$. Hence the only remaining possibility is that property (ii) for $k=0$ of Definition 5.2 is violated. Hence there exist $m \in \mathbb{N}, L \subset \subset V$ for a chart $(V, \varphi)$ in $X, L^{\prime} \subset \subset W$ for a vector bundle chart $(W, \Psi)$ in $F$ and sequences $\varepsilon_{j}<1 / j$, $p_{j} \in L \cap \underline{u_{\varepsilon_{j}}^{-1}}\left(L^{\prime}\right) \cap{\underline{v_{\varepsilon_{j}}}}^{-1}\left(L^{\prime}\right)$ with

$$
\left|\boldsymbol{\psi} \circ u_{\varepsilon}\left(p_{j}\right)-\boldsymbol{\psi} \circ v_{\varepsilon}\left(p_{j}\right)\right|>j \varepsilon_{j}^{m} .
$$

We set $p_{\varepsilon}:=p_{j}$ for $\varepsilon_{j+1}<\varepsilon \leq \varepsilon_{j}$. Then $\tilde{p}:=\left[\left(p_{\varepsilon}\right)_{\varepsilon}\right] \in \widetilde{X}_{c}$ and $u(\tilde{p}) \neq v(\tilde{p})$.

Finally, compositions can be carried out unrestrictedly:

5.6 Theorem. Let $u=\left[\left(u_{\varepsilon}\right)_{\varepsilon}\right] \in \mathcal{G}[X, Y], v=\left[\left(v_{\varepsilon}\right)_{\varepsilon}\right] \in \mathcal{G}^{h}[Y, G]$ and $w=$ $\left[\left(w_{\varepsilon}\right)_{\varepsilon}\right] \in \operatorname{Hom}_{\mathcal{G}}[G, H]$. Then $v \circ u:=\left[\left(v_{\varepsilon} \circ u_{\varepsilon}\right)_{\varepsilon}\right]$ and $w \circ v:=\left[\left(w_{\varepsilon} \circ v_{\varepsilon}\right)_{\varepsilon}\right]$ are well-defined elements of $\mathcal{G}^{h}[X, G]$ and $\mathcal{G}^{h}[Y, H]$, respectively. 
Proof. Moderateness of $\left(v_{\varepsilon} \circ u_{\varepsilon}\right)_{\varepsilon}$ and $\left(w_{\varepsilon} \circ v_{\varepsilon}\right)_{\varepsilon}$ follows in a straightforward way from the definitions. The respective classes are well-defined (i.e., independent of the representatives of $u, v, w$ ) by Theorems 3.5, 4.5 and 5.5 (ii).

We next demonstrate that for any $u \in \mathcal{G}[X, Y]$ the space $\operatorname{Hom}_{u}(E, F):=$ $\{v \in \operatorname{Hom}(E, F) \mid \underline{v}=u\}$ can naturally be endowed with the structure of an $\mathbb{R}$-vector space. Although the corresponding statement in the smooth case is self-evident, in general the representatives $\left(v_{\varepsilon}\right)_{\varepsilon},\left(v_{\varepsilon}^{\prime}\right)_{\varepsilon}$ of elements $v, v^{\prime}$ of $\operatorname{Hom}_{u}(E, F)$ need not project onto the same representative $\left(u_{\varepsilon}\right)_{\varepsilon}$ of $u=\underline{v}=$ $\underline{v^{\prime}} \in \mathcal{G}[X, Y]$, so that simple fiberwise addition is in general not possible. In order to handle this problem we employ the following result

5.7 Proposition. Let $u=\left[\left(u_{\varepsilon}\right)_{\varepsilon}\right] \in \mathcal{G}[X, Y]$ and $v \in \operatorname{Hom}_{u}(E, F)$. Then there exists a representative $\left(v_{\varepsilon}\right)_{\varepsilon}$ of $v$ such that $\underline{v_{\varepsilon}}=u_{\varepsilon}$ for all $\varepsilon \in I$.

Proof. We suppose that $X$ is connected for the moment, the modifications for the non-connected case are then obvious. By $\mu_{F}: \mathbb{R} \times F \rightarrow F$ we denote fiberwise scalar multiplication. Endow $Y$ with any Riemannian metric $h$ and cover $Y$ with $d_{h}$-balls $W_{\alpha}^{(r)}$ of radius $r$ such that $\left(W_{\alpha}^{(2 r)}, \Psi_{\alpha}\right)$ is a vector bundle atlas of $F$. Choose a partition of unity $\chi_{j}(j \in \mathbb{N})$ subordinate to the covering $\left(W_{\alpha}^{(r)}\right)_{\alpha}$ of $Y$ and let supp $\chi_{j} \subset \subset W_{\alpha_{j}}^{(r)}$ for $j \in \mathbb{N}$. Let $\left(\tilde{v}_{\varepsilon}\right)_{\varepsilon}$ be any representative of $v$. For any relatively compact open subset $V$ of $X$ there exists some $\varepsilon_{0}$ such that $\sup _{p \in V} d_{h}\left(\underline{\tilde{v}_{\varepsilon}}(p), u_{\varepsilon}(p)\right)<r$. Hence for $\varepsilon<\varepsilon_{0}$ and $p \in V, u_{\varepsilon}(p) \in \operatorname{supp}\left(\chi_{j}\right)$ implies $\pi_{Y}\left(\tilde{v}_{\varepsilon}(p)\right) \in \operatorname{dom}\left(\psi_{\alpha_{j}}\right)$ and we may define for $e \in \pi_{X}^{-1}(V)$ :

$$
v_{\varepsilon}(e):=\sum_{j \in \mathbb{N}} \mu_{F}\left(\chi_{j} \circ u_{\varepsilon} \circ \pi_{X}(e), \Psi_{\alpha_{j}}^{-1}\left(\psi_{\alpha_{j}}\left(u_{\varepsilon}\left(\pi_{X}(e)\right)\right), \psi_{\alpha_{j}}\left(\tilde{v}_{\varepsilon}(e)\right)\right)\right)
$$

Since

$$
\begin{aligned}
\tilde{v}_{\varepsilon}(e) & =\sum_{j \in \mathbb{N}} \mu_{F}\left(\chi_{j} \circ \underline{\tilde{v}_{\varepsilon}} \circ \pi_{X}(e), \tilde{v}_{\varepsilon}(e)\right) \\
& =\sum_{j \in \mathbb{N}} \mu_{F}\left(\chi_{j} \circ \underline{\tilde{v}_{\varepsilon}} \circ \pi_{X}(e), \Psi_{\alpha_{j}}^{-1}\left(\psi_{\alpha_{j}}\left(\underline{\tilde{v}_{\varepsilon}}\left(\pi_{X}(e)\right)\right), \boldsymbol{\psi}_{\alpha_{j}}\left(\tilde{v}_{\varepsilon}(e)\right)\right)\right)
\end{aligned}
$$

it follows from $v \in \operatorname{Hom}_{u}(E, F)$ and the definition of $\sim_{v b}$ that $\left(v_{\varepsilon}\right)_{\varepsilon}$ is a representative of $\left.v\right|_{V}$. Finally, to construct a global representative of $v$ with the claimed properties we first note that by our connectedness assumption $X$ is $\sigma$-compact, hence we may choose an exhaustive sequence $K_{m}(m \in \mathbb{N})$ of compact subsets of $X$ such that $K_{m} \subset \subset K_{m+1}^{\circ}$ for all $m$. By the above for each $m \in \mathbb{N}$ there exists $\varepsilon_{m}>0$ such that $v_{\varepsilon}$ as in (10) is defined on $K_{m}^{\circ}$ for $\varepsilon<\varepsilon_{m}$. According to [11], Lemma 10.3 there exists a smooth $f: X \rightarrow(0,1]$ such that $0<f(p) \leq \varepsilon_{n}$ for $p \in K_{n} \backslash K_{n-1}^{\circ}$. Thus by defining $v$ according to (10) for $(e, \varepsilon) \in\left\{(e, \varepsilon) \in E \times I \mid \varepsilon<f\left(\pi_{X}(e)\right)\right\}$ (and arbitrarily elsewhere) we obtain the desired global representative of $v$. 
5.8 Corollary. For any $u \in \mathcal{G}[X, Y], \operatorname{Hom}_{u}(E, F)$ is a vector space.

Proof. Fixing any representative $\left(u_{\varepsilon}\right)_{\varepsilon}$ of $u$, for any $v_{1}, v_{2} \in \operatorname{Hom}_{u}(E, F)$ we may choose representatives as in Proposition 5.7, so the claim follows.

By a similar reasoning it follows that in case $X=Y$ the space of strict generalized vector bundle homomorphisms $\operatorname{Hom}_{i d_{X}}(E, F)$ is naturally endowed with a vector space structure.

We finish this section demonstrating one of the consequences of the above result in the context of the induced covariant derivative of generalized vector fields on a curve introduced in [19], Sec. 5. Let $\alpha \in \mathcal{G}[J, X]$, where $J$ is an interval in $\mathbb{R}$ and let $\hat{g}$ be a generalized metric on $X$ (cf. Def. 3.4 in [19]) with generalized Levi Civita connection $\hat{D}$ (cf. [19], Def. 5.1 and Th. 5.2). For any generalized vector field $\xi$ on $\alpha$, i.e., $\xi=\left[\left(\xi_{\varepsilon}\right)_{\varepsilon}\right] \in \mathfrak{X}_{\mathcal{G}}(\alpha)$ the induced covariant derivative $\xi^{\prime}$ is defined componentwise, i.e., $\xi^{\prime}:=\left[\left(\xi_{\varepsilon}^{\prime}\right)_{\varepsilon}\right]$, where $\xi_{\varepsilon}^{\prime}$ is the classical induced covariant derivative with respect to a representative $\left(\hat{g}_{\varepsilon}\right)_{\varepsilon}$ of $\hat{g}$. Note that by [19], Th. 3.1 any generalized pseudo-Riemannian metric (on any relatively compact open set) has a representative which consists of classical pseudo-Riemannian metrics for $\varepsilon$ small enough. Now for $\xi, \eta \in \mathfrak{X}_{\mathcal{G}}(\alpha)$ we may define $\hat{g}(\xi, \eta)$ componentwise since by Proposition 5.7 we may choose representatives $\left(\xi_{\varepsilon}\right)_{\varepsilon}$ and $\left(\eta_{\varepsilon}\right)_{\varepsilon}$ of $\xi$ respectively $\eta$ with $\underline{\xi_{\varepsilon}}=\underline{\eta_{\varepsilon}}=\alpha_{\varepsilon}$ for some representative $\left(\alpha_{\varepsilon}\right)_{\varepsilon}$ of $\alpha$. Hence both sides of the equation

$$
\frac{d}{d t} \hat{g}(\xi, \eta)=\hat{g}\left(\xi^{\prime}, \eta\right)+\hat{g}\left(\xi, \eta^{\prime}\right)
$$

are well-defined. Moreover, equation (11) indeed holds by an application of the classical result (cf. e.g. [22], Prop 3.18) at the level of representatives.

\section{Coupled Calculus}

In all variants of Colombeau generalized functions taking values in a linear space compatibility with respect to the distributional setting is effected through the notion of association. We say an element $\left(u_{\varepsilon}\right)_{\varepsilon}$ of $\mathcal{E}_{M}(X)$ is associated to zero if $\int u_{\varepsilon} \nu \rightarrow 0$ for all compactly supported one-densities $\nu$ on $X$ (see [10], Sec. 2 ), i.e., if $u_{\varepsilon} \rightarrow 0$ weakly. If $\left(u_{\varepsilon}\right)_{\varepsilon} \in \mathcal{N}(X)$ then it is automatically associated to zero, so we may define a generalized function to be associated to zero if its representatives are. We say that two generalized functions are associated, $u \approx v$ if $u-v \approx 0$. The equivalence relation $\approx$ allows us to define the linear quotient space $\mathcal{G}(X) / \approx$. Equality in $\mathcal{G}(X)$ clearly implies association but not conversely (e.g., any power $H^{n}(n \geq 2)$ of the Heaviside function $H$ in $\mathcal{G}(\mathbb{R})$ is associated but not equal to $H)$.

We say that $u \in \mathcal{G}(X)$ admits $w \in \mathcal{D}^{\prime}(X)$ as a distributional shadow if

$$
\lim _{\varepsilon \rightarrow 0} \int u_{\varepsilon} \nu=w(\nu), \quad \forall \nu \in \Gamma_{c}(\operatorname{Vol}(X))
$$


The distributional shadow of $u$ is uniquely determined (if it exists). Thus although the embedding $\iota$ of $\mathcal{D}^{\prime}(X)$ into $\mathcal{G}(X)$ is not unique, one recovers a unique description of $\mathcal{D}^{\prime}(X)$ within the Colombeau algebra by working with $\iota\left(D^{\prime}(X)\right) / \approx$.

To extend the concept of association to the context of generalized functions taking values in a smooth manifold we would need a "diffeomorphism invariant characterization" of weak convergence. Based on the results of this paper it is natural to aim at a notion of association defined by $\left[\left(u_{\varepsilon}\right)_{\varepsilon}\right] \approx\left[\left(v_{\varepsilon}\right)_{\varepsilon}\right] \in$ $\mathcal{G}[X, Y]: \Leftrightarrow\left[\left(f \circ u_{\varepsilon}-f \circ v_{\varepsilon}\right)_{\varepsilon}\right] \approx 0 \in \mathcal{G}(X) \forall f \in \mathcal{C}^{\infty}(Y)$. However, such a construction does not reproduce the standard concept of association in the case that $Y$ is a vector space since weak convergence cannot be characterized by composition with smooth functions. To see this we first note that by [21], Ex. 10.6, we may choose $d_{1}, d_{2} \in \mathcal{G}(\mathbb{R})$, both associated to $\delta$ such that $d_{i}^{2} \approx c_{i} \delta$ $(i=1,2)$ with $c_{i} \in \mathbb{C}$ and $c_{1} \neq c_{2}$. Hence $d_{1} \approx d_{2}$ but $f \circ d_{1} \not \approx f \circ d_{2}$ for $f=x \mapsto x^{2}$.

On the other hand the notion of $k$-association in $\mathcal{G}(X)$ as introduced in [18], Sec. 5 can be generalized to the present setting using composition with smooth functions.

\subsection{Definition.}

(i) Let $u=\left[\left(u_{\varepsilon}\right)_{\varepsilon}\right]$, $v=\left[\left(v_{\varepsilon}\right)_{\varepsilon}\right] \in \mathcal{G}[X, Y]$. We call $u$ and $v k$-associated $(0 \leq k \leq \infty)$ with each other $\left(u \approx_{k} v\right)$ if $f \circ u \approx_{k} f \circ v \in \mathcal{G}(X)$ for all $f \in \mathcal{C}^{\infty}(Y)$.

(ii) We say that $u=\left[\left(u_{\varepsilon}\right)_{\varepsilon}\right] \in \mathcal{G}[X, Y]$ admits $g: X \rightarrow Y$ as $\mathcal{C}^{k}$-associated function $\left(u \approx_{k} g\right.$ ) if $f \circ u \approx_{k} f \circ g \in \mathcal{G}(X)$ for all $f \in \mathcal{C}^{\infty}(Y)$.

Analogously to the case of $\mathcal{G}(X)[18]$, Def. 1 (ii) if $u \in \mathcal{G}[X, Y]$ is $k$-associated to a function $g$, then $g \in \mathcal{C}^{k}(X, Y)$. Furthermore if $u$ admits a $k$-associated function at all, then the latter is unique.

If $Y=\mathbb{R}^{n}$ the notion defined in (i) above coincides with the one of [18], Def. $1(\mathrm{i})$. To see this take $\left[\left(u_{\varepsilon}\right)_{\varepsilon}\right] \approx_{k}\left[\left(v_{\varepsilon}\right)_{\varepsilon}\right] \in \mathcal{G}(X)^{n}$. Since $\left\|u_{\varepsilon}-v_{\varepsilon}\right\| \rightarrow 0$ on compact sets the estimates on $\left\|f\left(u_{\varepsilon}(x)\right)-f\left(v_{\varepsilon}(x)\right)\right\|$ follow from [15], Lemma 5. The derivatives can be estimated in the same way. We have the following characterization of 0 -equivalence.

6.2 Proposition. Let $\left(u_{\varepsilon}\right)_{\varepsilon},\left(v_{\varepsilon}\right)_{\varepsilon} \in \mathcal{G}[X, Y]$. The following statements are equivalent:

(i) $\left(u_{\varepsilon}\right)_{\varepsilon} \approx_{0}\left(v_{\varepsilon}\right)_{\varepsilon}$.

(ii) For every Riemannian metric $h$ on $Y$ and every $K \subset \subset X$,

$$
\sup _{p \in K} d_{h}\left(u_{\varepsilon}(p), v_{\varepsilon}(p)\right) \rightarrow 0 \quad(\varepsilon \rightarrow 0) .
$$

Proof. This follows in complete analogy to the proof of the equivalence of (iii) and (iv) of Theorem 3.3 . 


\subsection{Examples.}

(i) By Theorem 3.3 two elements $\left(u_{\varepsilon}\right)_{\varepsilon},\left(v_{\varepsilon}\right)_{\varepsilon}$ of $\mathcal{G}[\mathbb{R}, \mathbb{R}]$ are equivalent if and only if for each $K \subset \subset \mathbb{R} \forall m \in \mathbb{N} \sup _{x \in K}\left|u_{\varepsilon}(x)-v_{\varepsilon}(x)\right|=O\left(\varepsilon^{m}\right)$. Hence the classes of $u_{\varepsilon}(x)=\varepsilon x, v_{\varepsilon}(x)=\varepsilon^{2} x^{2}$ in $\mathcal{G}[\mathbb{R}, \mathbb{R}]$ are different, but are easily seen to be 0 -associated.

(ii) In [17], solutions of the geodesic equation for a singular spacetime metric with line-element

$$
d s^{2}=\delta(u) f(x, y) d u^{2}-d u d v+d x^{2}+d y^{2}
$$

are calculated. Based on Theorem 1 of that paper it is shown in the final section of 19] that the $x$-component of the unique solution of this ODE is c-bounded, hence can be regarded as a manifold-valued generalized function. Furthermore, from [17, Theorem 3 it follows that it converges locally uniformly (hence by Proposition 6.2 is 0 -associated) to a kink function. This corresponds to the physical expectation of broken geodesics of the singular metric (12).

\section{References}

[1] Aragona, J., Biagioni, H. A. Intrinsic definition of the Colombeau algebra of generalized functions. Analysis Mathematica, 17:75-132, 1991.

[2] Balasin, H. Distributional energy-momentum tensor of the extended Kerr geometry. Class. Quant. Grav., 14:3353-3362, 1997.

[3] Clarke, C. J. S., Vickers, J. A., Wilson, J. P. Generalised functions and distributional curvature of cosmic strings. Class. Quant. Grav., 13:24852498, 1996.

[4] Colombeau, J. F. New Generalized Functions and Multiplication of Distributions. North Holland, Amsterdam, 1984.

[5] Colombeau, J. F. Elementary Introduction to New Generalized Functions. North Holland, Amsterdam, 1985.

[6] Colombeau, J. F., Meril, A. Generalized functions and multiplication of distributions on $\mathcal{C}^{\infty}$ manifolds. J. Math. Anal. Appl., 186:357-364, 1994.

[7] Dapić, N., Kunzinger, M., Pilipović, S. Symmetry group analysis of weak solutions. Proc. London Math. Soc., 84(3):686-710, 2002.

[8] Dapić, N., Pilipović, S. Microlocal analysis of Colombeau's generalized functions on a manifold. Indag. Math. (N.S.), 7:293-309, 1996.

[9] De Rham, G. Differentiable Manifolds, of Grundlehren der mathematischen Wissenschaften 266. Springer, Berlin, 1984. 
[10] De Roever, J. W., Damsma, M. Colombeau algebras on a $\mathcal{C}^{\infty}$-manifold. Indag. Mathem., N.S., 2(3), 1991.

[11] Grosser, M., Farkas, E., Kunzinger, M., Steinbauer, R. On the foundations of nonlinear generalized functions I, II. Mem. Amer. Math. Soc., 153(729), 2001.

[12] Grosser, M., Kunzinger, M., Oberguggenberger, M., Steinbauer, R. Geometric Theory of Generalized Functions with Applications to Relativity, Mathematics and its Applications 537. Kluwer, 2001.

[13] Grosser, M., Kunzinger, M., Steinbauer, R., Vickers, J. A global theory of algebras of generalized functions. Adv. Math., 166(1):179-206, 2002.

[14] Jelínek, J. An intrinsic definition of the Colombeau generalized functions. Comment. Math. Univ. Carolinae, 40:71-95, 1999.

[15] Kunzinger, M. Generalized functions valued in a smooth manifold. Monatsh. Math., to appear. Available electronically at http://arxiv.org/ abs/math.FA/0107051.

[16] Kunzinger, M., Oberguggenberger, M. Group analysis of differential equations and generalized functions. SIAM J. Math. Anal., 31(6):1192-1213, 2000.

[17] Kunzinger, M., Steinbauer, R. A rigorous solution concept for geodesic and geodesic deviation equations in impulsive gravitational waves. J. Math. Phys., 40:1479-1489, 1999.

[18] Kunzinger, M., Steinbauer, R. Foundations of a nonlinear distributional geometry. Acta Appl. Math., 71:179-206, 2002.

[19] Kunzinger, M., Steinbauer, R. Generalized pseudo-Riemannian geometry. Trans. Amer. Math. Soc., to appear. Available electronically at http://arxiv.org/abs/math.FA/0107057.

[20] Marsden, J. E. Generalized Hamiltonian mechanics. Arch. Rat. Mech. Anal., 28(4):323-361, 1968.

[21] Oberguggenberger, M. Multiplication of Distributions and Applications to Partial Differential Equations, Pitman Research Notes in Mathematics 259. Longman, Harlow, U.K., 1992.

[22] O'Neill, B. Semi-Riemannian Geometry (With Applications to Relativity). Academic Press, New York, 1983.

[23] Vickers, J. A. Nonlinear generalized functions in general relativity. In Grosser, M., Hörmann, G., Kunzinger, M., Oberguggenberger, M., ed., Nonlinear Theory of Generalized Functions, CRC Research Notes in Mathematics 401, 275-290, Boca Raton, 1999. 
[24] Wilson, J. P. Distributional curvature of time dependent cosmic strings. Class. Quantum Grav., 14:3337-3351, 1997. 